\title{
Material parameter identification of unidirectional fiber-reinforced composites
}

Received: 13 August 2020 / Accepted: 21 January 2021 / Published online: 16 February 2021

(C) The Author(s) 2021

\begin{abstract}
In this article, several aspects of material parameter identification are addressed. We compare several methods to identify material parameters of a constitutive model for small strain, linear elastic transverse isotropy based on experimental data of specimens made from composite plates. These approaches range from identifying the five material parameters from purely analytical considerations to the fully numerical identification on the basis of finite elements and various data provided by digital image correlation (DIC). The underlying experimental tests range from purely uniaxial tensile tests with varying fiber orientation to shear and compression tests. A specific measuring instrument has been developed for the latter tests to obtain unique material parameters-motivated by the concept of local identifiability. Besides, we compare the numerical differentiation, which is the common procedure in parameter identification, with the fully analytical derivation of sensitivities within the DIC/FEM approach.
\end{abstract}

Keywords Material parameter identification · Least-square method · Finite elements · Transverse isotropy · Digital image correlation

\section{Introduction}

One of the simplest models incorporating anisotropy is represented by transverse isotropy, where the material has one pronounced orientation. Here, we restrict ourselves to the case of purely linear elastic material models. This can be formulated by an invariant theory, for example $[25,42,44]$, offering the possibility to also treat physically and geometrically nonlinear behavior. The restriction to linear behavior can be obtained by linearizing the equations so that merely quantities depending linearly on the strain tensor can be obtained. These purely tensorial formulations, which have the advantage of being independent on the choice of the coordinate system, can be adapted to curvilinear problems, [47] and [15], or to Cartesian coordinates. In the latter case, it is common to reformulate tensorial expressions into matrix equations, where either the Voigt or the Kelvin notation can be applied, see [46]. The components of the stress and strain tensors are assembled in $(6 \times 1)$ column vectors, and the $(6 \times 6)$ elasticity matrix connects both quantities. This symmetric matrix contains-in the case of transverse isotropy - 5 independent material parameters, which have to be determined by experimental tests. Unfortunately, it is not really clear, which tests are necessary to determine the parameters more or less uniquely. One approach is discussed in Christensen [8], where several experiments are proposed. However, one of these experiments is very difficult to carry out or almost impossible to implement due to the boundary conditions. These tests have the advantage that they describe homogeneous deformations so that —on the level of matrix representation of the constitutive model - the material parameters are determinable.

S. Hartmann ( $\varangle)$ - R. R. Gilbert · A. K. Marghzar · C. Leistner · P. K. Dileep

Institute of Applied Mechanics, Clausthal University of Technology, Adolph-Roemer-Str. 2a, 38678 Clausthal-Zellerfeld,

Germany

E-mail: stefan.hartmann@tu-clausthal.de 
Digital image correlation (DIC) systems are a more modern approach, where full-field measurements of the displacements or strains on a subregion of the surface of a specimen are chosen to compare the data with finite element simulations, [2,31]. Further papers following this concept are [4,9,26-28,38] using gradient-based optimization methods. Here, a least-square method is chosen to minimize the residual between the experimental data and numerical results. For a gradient-free scheme see [17], where the numerical algorithm of Powell $[35,36]$ is chosen, or $[23,24]$ applying a neural network approach. Further applications are shown, for example, in $[6,18,30,37]$, where indentation tests are applied. The theoretical basis of parameter identification using finite elements and constitutive models of evolutionary type is discussed in [13] focusing on the relationship to numerical mathematics.

In this article, we discuss the material parameter identification process for transverse isotropy using two approaches-first assuming homogeneous deformations and then, secondly, inhomogeneous ones. For this purpose, we carry out uniaxial tensile tests on fiber-reinforced specimens under three different fiber orientations, a shear test, and a particular compression test. Furthermore, the concept of local identifiability, see [3,5], is transferred to the investigation under consideration. For purely elastic materials, see the discussions in $[14,20]$. Several identification procedures and the reliability of the parameters obtained are discussed.

The notation in use is defined in the following manner: Geometrical vectors are symbolized by $\vec{a}$, secondorder tensors $\mathbf{A}$ by boldfaced Roman letters, and calligraphic letters $\mathcal{A}$ define fourth-order tensors. Furthermore, we introduce matrices at global level symbolized by boldfaced italic letters $\boldsymbol{A}$ and matrices on local level (Gauss point level) using boldfaced Roman letters $\mathbf{A}$.

\section{Experimental and model considerations}

\subsection{Model of transverse isotropy}

A common model to describe the material behavior of composites with unidirectional fiber orientation is represented by linear elastic transverse isotropy. One model representation is given in [44]. For the case of small strains, this model reads

$$
\mathbf{T}=\left(\Lambda \mathrm{I}_{\mathbf{E}}+\alpha \mathrm{IV}_{\mathbf{E}}\right) \mathbf{I}+2 \mu_{T} \mathbf{E}+\left(\alpha \mathrm{I}_{\mathbf{E}}+\beta \mathrm{IV}_{\mathbf{E}}\right) \mathbf{M}+2\left(\mu_{L}-\mu_{T}\right)(\mathbf{E M}+\mathbf{M E}),
$$

or using $\mathbf{T}=\mathcal{C} \mathbf{E}$, it is expressed by the fourth-order elasticity tensor

$$
\begin{aligned}
\mathcal{C} & =\Lambda \mathbf{I} \otimes \mathbf{I}+2 \mu_{T} \mathcal{I}+\alpha[\mathbf{I} \otimes \mathbf{M}+\mathbf{M} \otimes \mathbf{I}]+\beta \mathbf{M} \otimes \mathbf{M}+2\left(\mu_{L}-\mu_{T}\right)[\mathbf{I} \otimes \mathbf{M}+\mathbf{M} \otimes \mathbf{I}]^{T_{23}} . \\
\mathbf{E}(\mathbf{x}, t) & =\frac{1}{2}\left(\operatorname{grad} \mathbf{u}(\mathbf{x}, t)+\operatorname{grad}^{T} \mathbf{u}(\mathbf{x}, t)\right)
\end{aligned}
$$

defines the linearized Green strain tensor, and

$$
\mathrm{I}_{\mathbf{E}}=\operatorname{tr} \mathbf{E}, \quad \mathrm{II}_{\mathbf{E}}=\operatorname{tr} \mathbf{E}^{2}, \quad \mathrm{III}_{\mathbf{E}}=\operatorname{tr} \mathbf{E}^{3}, \quad \mathrm{IV}_{\mathbf{E}}=\operatorname{tr}(\mathbf{E M})=\mathbf{E} \cdot \mathbf{M}, \quad \mathrm{V}_{\mathbf{E}}=\operatorname{tr}\left(\mathbf{E}^{2} \mathbf{M}\right)=\mathbf{E}^{2} \cdot \mathbf{M}
$$

represent the invariants concerning transverse isotropy. $\mathbf{M}=\mathbf{a} \otimes \mathbf{a}$ symbolizes the structural tensor, where a defines the fiber direction. $\mathcal{I}=[\mathbf{I} \otimes \mathbf{I}]^{T_{23}}=\delta_{i k} \delta_{j l} \mathbf{e}_{i} \otimes \mathbf{e}_{j} \otimes \mathbf{e}_{k} \otimes \mathbf{e}_{l}$ is the fourth-order identity tensor, $\mathbf{A}=\mathcal{I} \mathbf{A}$. The symbol $\mathcal{A}^{T_{23}}$ implies the transposition of second and third index of the fourth-order tensor $\mathcal{A}$, i.e., for $\mathcal{A}=a_{i j k l} \mathbf{e}_{i} \otimes \mathbf{e}_{j} \otimes \mathbf{e}_{k} \otimes \mathbf{e}_{l}$ we obtain $\mathcal{A}^{T_{23}}=a_{i k j l} \mathbf{e}_{i} \otimes \mathbf{e}_{j} \otimes \mathbf{e}_{k} \otimes \mathbf{e}_{l}$.

One particular property of the model is its additive decomposition of the stress state $\mathbf{T}=\mathbf{T}_{\text {iso }}+\mathbf{T}_{\text {ani }}$ into an "isotropic" and an "anisotropic" part

$$
\begin{aligned}
& \mathbf{T}_{\text {iso }}=\Lambda \mathrm{I}_{\mathbf{E}} \mathbf{I}+2 \mu_{T} \mathbf{E}, \\
& \mathbf{T}_{\text {ani }}=\alpha \mathrm{IV}_{\mathbf{E}} \mathbf{I}+\left(\alpha \mathrm{I}_{\mathbf{E}}+\beta \mathrm{IV}_{\mathbf{E}}\right) \mathbf{M}+2\left(\mu_{L}-\mu_{T}\right)(\mathbf{E M}+\mathbf{M E}),
\end{aligned}
$$

implying the fourth-order tensors $\mathcal{C}=\mathcal{C}_{\text {iso }}+\mathcal{C}_{\text {ani }}$,

$$
\begin{aligned}
& \mathcal{C}_{\text {iso }}=\Lambda \mathbf{I} \otimes \mathbf{I}+2 \mu_{T} \mathcal{I}, \\
& \mathcal{C}_{\text {ani }}=\alpha[\mathbf{I} \otimes \mathbf{M}+\mathbf{M} \otimes \mathbf{I}]+\beta \mathbf{M} \otimes \mathbf{M}+2\left(\mu_{L}-\mu_{T}\right)[\mathbf{I} \otimes \mathbf{M}+\mathbf{M} \otimes \mathbf{I}]^{T_{23}},
\end{aligned}
$$




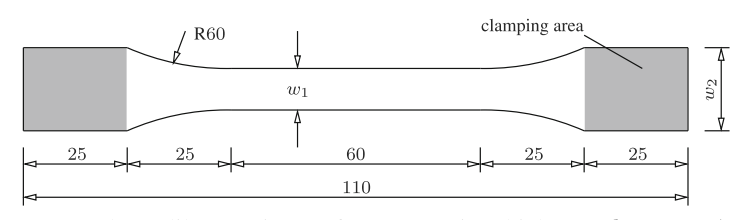

(a) Dog-bone-like specimens for pure resin (thickness $d=4.1 \pm$ $0.1 \mathrm{~mm}$, width $w_{1}=10.18 \pm 0.27 \mathrm{~mm}$ and $w_{2}=20.09 \pm$ $0.33 \mathrm{~mm}$ )

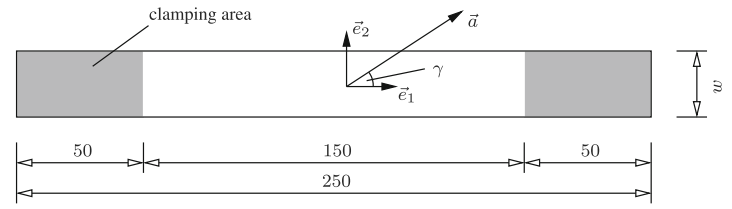

(b) Specimens for reinforced composites (thickness $d=2.4 \mathrm{~mm}$, width $w=25 \mathrm{~mm}$ for $45^{\circ}$ and $90^{\circ}$, and $w=15 \mathrm{~mm}$ for $0^{\circ}$ )

Fig. 1 Specimens (dimensions in $\mathrm{mm}$ )

see comments on minor and major symmetries of the fourth-order elasticity tensor in Appendix B. In this sense, $\Lambda$ and $\mu_{T}$ represent the Lame constants depending on the Young's modulus $E$ and the Poisson's number $v$ by

$$
\Lambda=\frac{v E}{(1+v)(1-2 v)}, \quad \mu_{T}=\frac{E}{2(1+v)},
$$

i.e., we can reformulate the fourth-order elasticity tensor $\mathcal{C}_{\text {iso }}$ by

$$
\mathcal{C}_{\text {iso }}=\frac{E}{1+v} \mathcal{I}+\frac{E v}{(1+v)(1-2 v)} \mathbf{I} \otimes \mathbf{I}
$$

as well. The Voigt notation of the fourth-order elasticity tensor is summarized in Appendix A.

\subsection{Experiments at pure resin and unidirectional composite specimens}

In the following, we perform uniaxial tensile tests on pure resin specimens, uniaxial tensile experiments on specimens with unidirectional fiber reinforcement in three directions $\left(\gamma=0^{\circ}, 45^{\circ}, 90^{\circ}\right)$, a shear test, and a particular compression test. We choose glass fibers (Young's modulus $E_{f}=73,000 \mathrm{MPa}$, Poisson's number $v=0.22$ ) embedded in an epoxy resin RIMR135 with a curing agent RIMH1366 and a volume fraction of approximately 55\%. For the reinforcement, endless E-glass fibers HP-U400E are drawn on from HP-Textiles $\mathrm{GmbH}$. The fiber diameters are $13-24 \mu \mathrm{m}$, which are bundled to rovings of 4-7 $\mathrm{mm}$. The rovings are connected by binding threads (for a picture of the specimens, see [21]). The distance of the fiber bundles is around 0$1 \mathrm{~mm}$. We use the double-camera system ARAMIS 5M in connection with the software ARAMIS Professional 2017 from GOM GmbH, Brunswick, Germany.

\subsubsection{Tensile tests}

In each test, the displacement of the machine's traverse is prescribed linearly, the forces are recorded, and the deformation field is measured in the center region of the specimens using a DIC system (except for the compression test, where no optical access is possible). The geometries of the tensile specimens are shown in Fig. 1 . The traverse displacement is prescribed by $u(t) \mathbf{e}_{1}$ with $u(t)=\dot{u} t, \dot{u}=0.025 \mathrm{~mm} \mathrm{~s}^{-1}$. We measure the reaction force-signal of the testing machine's force gauge - and the surface displacements in the center region of the specimens. For each tensile problem-pure plane resin (dog-bone-like specimens), and flat fiber-reinforced specimens for $\gamma=0^{\circ}, 45^{\circ}, 90^{\circ}$, and $\gamma=90^{\circ}$-five tests are performed.

Exemplarily, the axial strain distribution of the tensile specimen of Fig. 1b is shown in Fig. 2. To minimize rigid body displacements resulting from the testing machine's flexibility, two arms are directly fixed (locallyhere, at the right-hand side with a ruler) at the clamps, and the measured displacements are modified relative to the arms.

One interesting effect is that the matrix material between the fiber rovings deforms more than in the roving region. Thus, a highly scattered strain distribution is present. However, we follow the common assumption of a homogeneously distributed material property. Since we do not assume a heterogeneous material, model (1) is assumed to be sufficient. It is well known that a homogeneous stress state is impossible for $\gamma=45^{\circ}$ since there is a coupling between both axial and lateral strains with shear, which is numerically demonstrated in [1]. Nevertheless, we show the load-deflection curves for the three tests $\gamma=0^{\circ}, 45^{\circ}, 90^{\circ}$, see Fig. $3 \mathrm{~b}$. Using a DIC system, we measure the surface displacements and surface strains in a subregion of the specimen. (The results are used for parameter identification later on). 


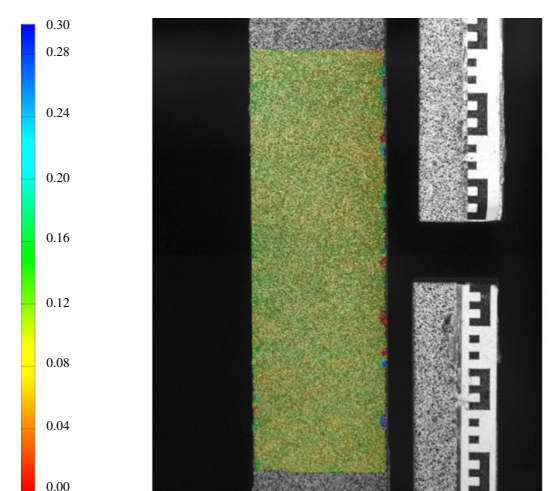

(a) Legend (b) $\gamma=0^{\circ}$

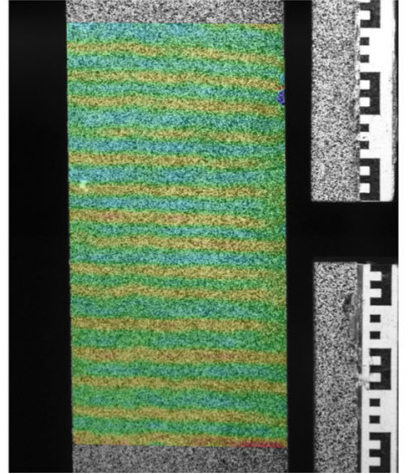

(c) $\gamma=90^{\circ}$

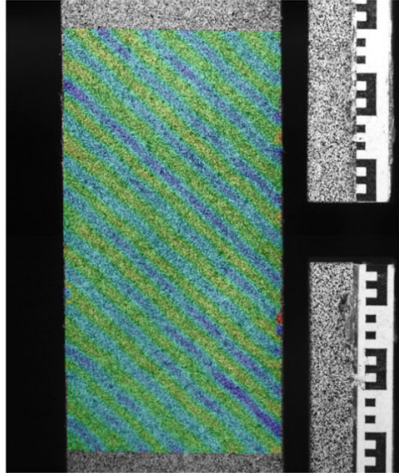

(d) $\gamma=45^{\circ}$

Fig. 2 DIC information of axial strains $\varepsilon_{11}$

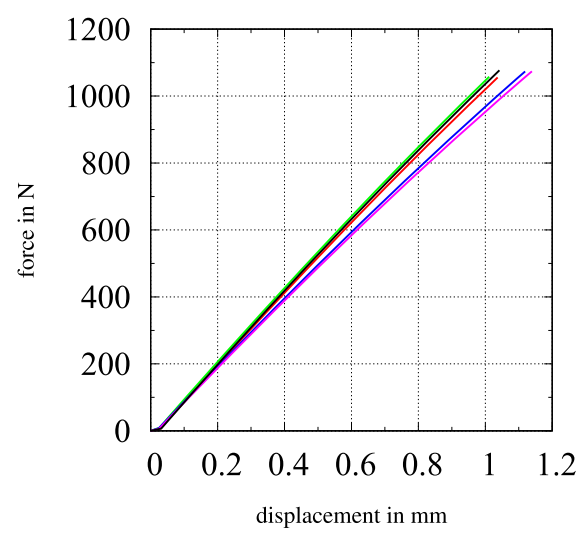

(a) Pure resin

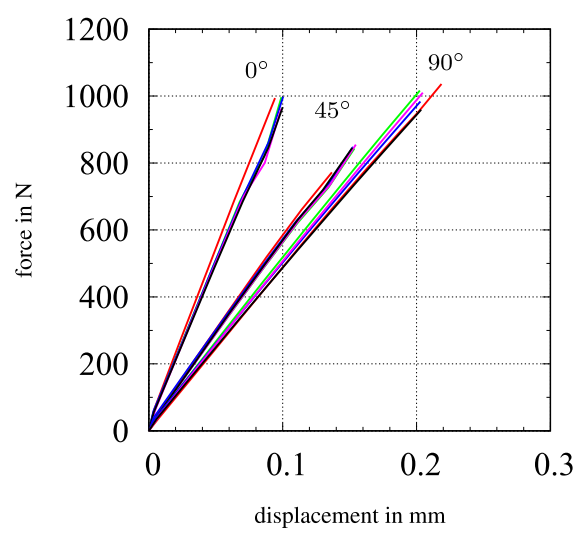

(b) Fiber-reinforced composite

Fig. 3 Load-displacement curves (traverse displacement) of pure resin material and various fiber orientations $\gamma=0,45,90^{\circ}$ (for each experiment five tests are performed)

\subsubsection{Shear tests}

Additionally, shear tests at specimens with $\gamma=90^{\circ}$ are performed using the shear tool proposed in [43]. Figure 4a shows the shear device of the three-rail shear tool is shown, whereas the results in Fig. 4c are required in the parameter identification process.

\subsubsection{Compression tests}

For the purpose of parameter identification, we built a compression device where an axial, compressive load can be applied, and the lateral stresses are measured, see Fig. 5a. In other words, we assume the stress and strain state

$$
\mathbf{T}=\left[\begin{array}{ccc}
\sigma_{11} & 0 & 0 \\
0 & \sigma_{22} & 0 \\
0 & 0 & \sigma_{33}
\end{array}\right], \quad \mathbf{E}=\left[\begin{array}{ccc}
0 & 0 & 0 \\
0 & 0 & 0 \\
0 & 0 & \varepsilon_{33}
\end{array}\right]
$$

We prescribe the axial strains $\varepsilon_{33}$ and measure both $\sigma_{11}$ and $\sigma_{22}$ by the force gauges in the compression tool as well as the axial stresses $\sigma_{33}$ by the testing machine's force gauge, see Fig. $5 \mathrm{~b}$. As it is common, the axial deformation was calibrated using the testing machine's stiffness, and several pretests with homogeneous materials were performed to find out whether the measuring system provides sufficient accuracy. For this, we chose aluminum and rubber material. Similarly to the lateral strain determination procedures in classical tensile tests, where the lateral strains are very small, the lateral force measurement in the compression test is highly sensitive due to geometrical imperfections of the specimens itself. Figure $6 \mathrm{~b}$, c shows the response of 


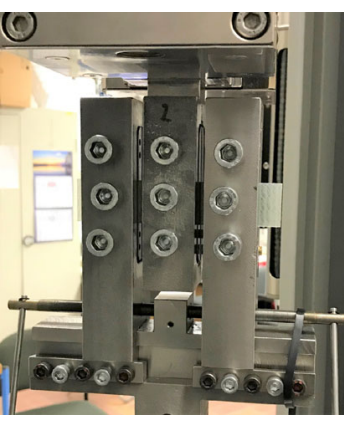

(a) Shear device

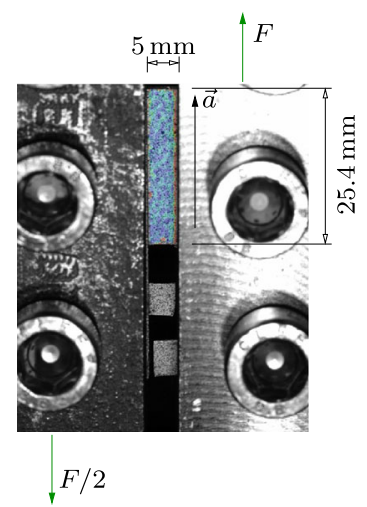

(b) Representative maximum strain image

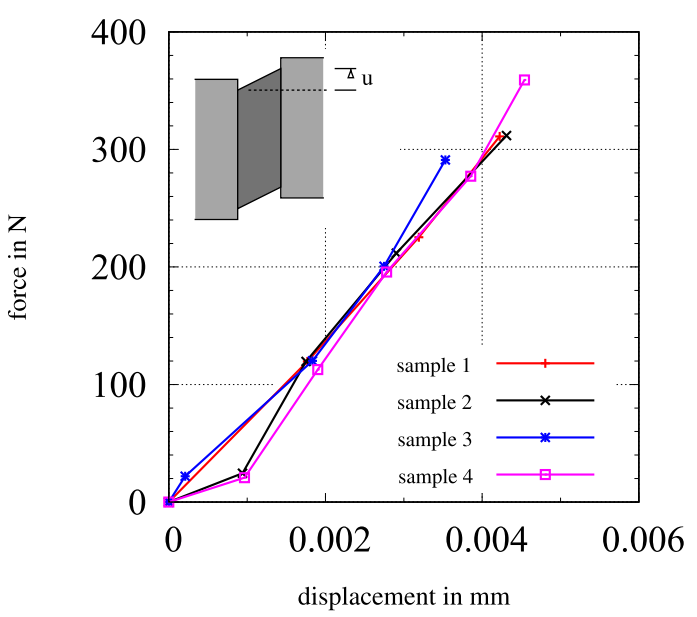

(c) Load-displacement curves of shear test (displacements of the testing machine - center rail clamp)

Fig. 4 Three-rail shear tool

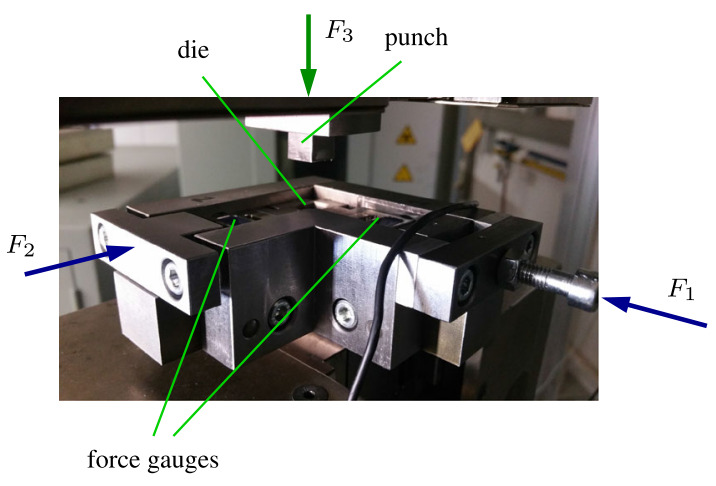

(a) Compression device

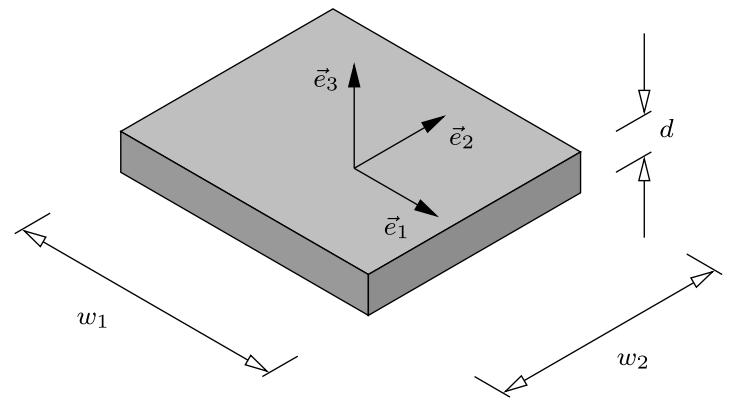

(b) Coordinate system of the sample geometry $\left(w_{1}=w_{2}=\right.$ $12 \mathrm{~mm}, d=2.4 \mathrm{~mm}$

Fig. 5 Compression device and chosen coordinate system

the lateral stresses resulting from the given strains $\varepsilon_{33}$ (and stresses $\sigma_{33}$ ), see Fig. 6a. The lubricant used for the compression testing is CRC SILICONE IND (synthetic oil spray) to reduce the friction, and the samples were brought to their final shape with a milling cutter. Unfortunately, this leads to melting of the material on the surface if the coolant is left away. Another reason for the dispersion can be the width of the samples used (12 mm), which is small in relation to the fiber bundle width of 4-7 mm. However, since this does not lead to a significant effect on the identification of the parameters in the averaged sense (compare later the results of the different approaches to identification), the experimental results are used in this form. Nevertheless, there is a need for further investigation for the future.

\section{Parameter identification of transverse isotropic material}

\subsection{Basics of parameter identification}

Since we are interested in the application of the least-square method, the exact number of experimental data for the material parameter identification process must be determined. In each experiment $\hat{E}, \hat{E}=1, \ldots, n_{\exp }$, we have the experimental data $\boldsymbol{d}^{(\hat{E})} \in \mathbb{R}^{n_{\exp }^{(\hat{E})}}$, with $n_{\exp }^{(\hat{E})}=n_{\mathrm{N}}^{(\hat{E})} n_{\mathrm{d}}^{(\hat{E})} \cdot n_{\mathrm{d}}^{(\hat{E})}$ are commonly more than several thousand (spatial) entries for DIC data, and a reaction force of the testing machine if there is a displacement control. In each experiment, we have $n_{\mathrm{N}}^{(\hat{E})}$ load steps (sampling times), i.e., evaluation times $t_{n}$, 


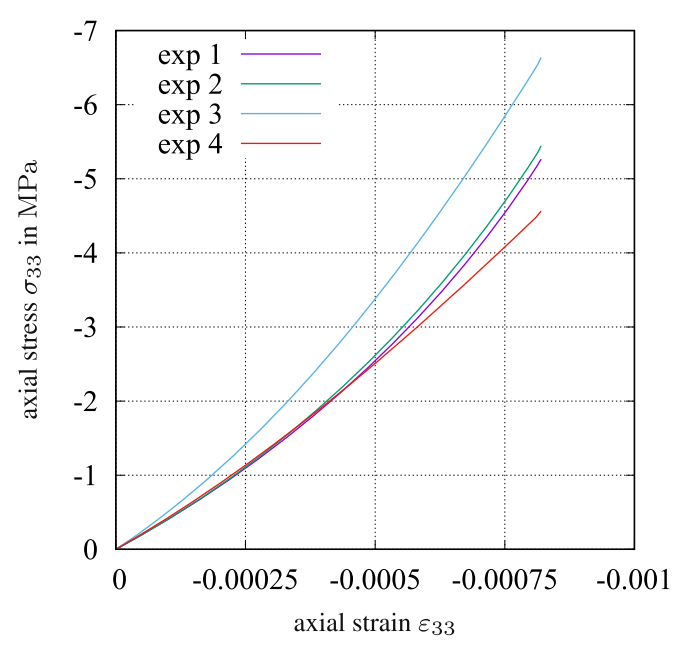

(a) Axial stress-strain information

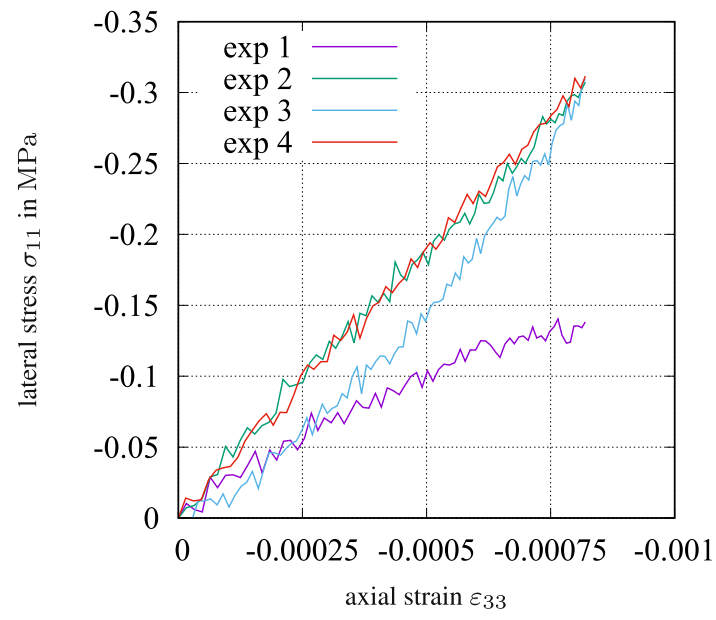

(b) Lateral stresses in $\vec{e}_{1}$ direction

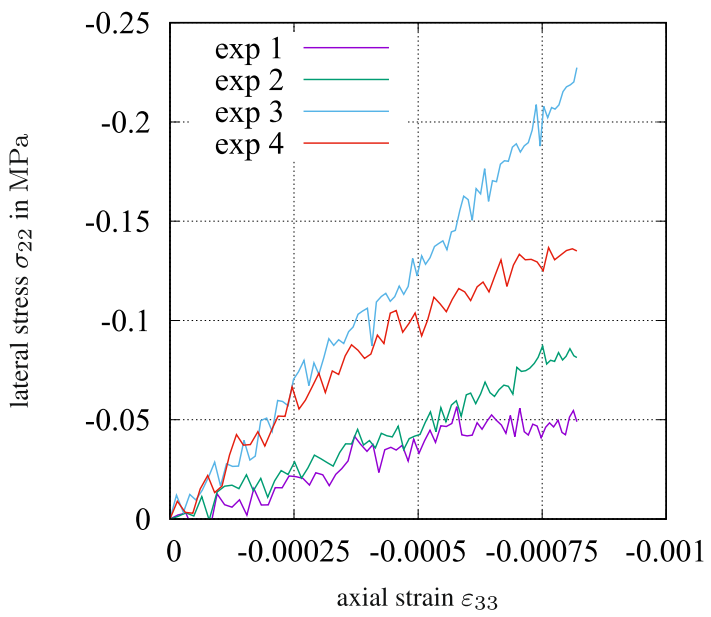

(c) Lateral stresses in $\vec{e}_{2}$ direction

Fig. 6 Experimental data of the compression tests $\left(\mathbf{a}=\mathbf{e}_{1}\right)$

$n=0, \ldots, n_{\mathrm{N}}^{(\hat{E})}$. In other words, we have to assemble the data $\boldsymbol{d}_{n}^{(\hat{E})} \in \mathbb{R}^{n_{\mathrm{d}}^{(\hat{E})}}$ at each evaluation time $t_{n}$ into the vector $\boldsymbol{d}^{(\hat{E}) T}=\left\{\boldsymbol{d}_{0}^{(\hat{E}) T}, \boldsymbol{d}_{1}^{(\hat{E}) T}, \ldots, \boldsymbol{d}_{n_{\mathrm{N}}^{(\hat{E})}}^{(\hat{E}) T}\right\}$. Accordingly, we compile all tests into a large vector $\boldsymbol{d}^{T}=\left\{\boldsymbol{d}^{(1) T}, \boldsymbol{d}^{(2) T}, \ldots, \boldsymbol{d}^{\left(n_{\exp }\right) T}\right\}$. The total number of experimental data is defined by $n_{\mathrm{D}}=\sum_{\hat{E}=1}^{n_{\exp }} n_{\exp }^{(\hat{E})}$. The experiments might be totally different or they are the same experiments performed multiple times. The data sets in each experiment are either displacements or some strain measures (strains in a particular direction maximum, principal strains, etc.) at particular points provided by a digital image correlation system, and the force gauge's information of the testing machine.

On the other hand, we have simulation data by means of finite elements, i.e., nodal displacements (or a strain measure) and resulting forces, which are evaluated at other points for each experiment. Thus, a linear interpolation in time to the experimental data at $t_{n}$ is chosen. In the case of linear problems, there are two possible approaches: Either the experimental data, where we have different increasing loading steps, are interpolated in advance before treating parameter identification-in which we obtain only one experimental data point per load step-or we consider all the data in the identification process for each loading step, yielding much more data to be considered in our identification procedure. 
In our case, the DIC region is smaller than the FEM region, i.e., only a subset of all nodal displacements $\boldsymbol{u}_{n}^{(\hat{E})} \in \mathbb{R}^{n_{\mathrm{u}}^{(\hat{E})}}$ of the finite element simulation (or strain measures at particular points of experiment $\hat{E}$ ) can be compared to the DIC data, $\tilde{\boldsymbol{u}}_{n}^{(\hat{E})}=\tilde{\boldsymbol{M}}^{(\hat{E})} \boldsymbol{u}_{n}^{(\hat{E})}, \tilde{\boldsymbol{u}}_{n}^{(\hat{E})} \in \mathbb{R}^{\tilde{n}_{\mathrm{u}}^{(\hat{E})}}$. The matrix $\tilde{\boldsymbol{M}}^{(\hat{E})}$ extracts only the necessary nodal displacements. There must be a spatial interpolation of the experimental data $\boldsymbol{d}_{n}^{(\hat{E})}$ to locations, where the numerical data $\tilde{\boldsymbol{u}}_{n}^{(\hat{E})}$ are provided (or the other way round). In this contribution, we project the FEM data onto the DIC data. Thus, an interpolation scheme is required. An essential drawback is that neither commercial DIC programs nor FEM programs provide displacements or strains at arbitrary points within the region under consideration. Here, we draw on the triangulation concept proposed in [16] and apply it to each surface displacement information (DIC and FEM), i.e., both the DIC and the FEM data are interpolated and the strains (maximum and minimum strains, etc.) can be computed. Thus, we choose a triangulation concept instead of the shape functions of the particular finite element formulation, in order to be as flexible as possible (which is applicable to commercial finite element programs as well). Additionally, we need the resulting reaction force of the finite element simulation

$$
F_{\mathrm{FEM} n}^{(\hat{E})}=\overline{\boldsymbol{M}}^{(\hat{E}) T} \boldsymbol{p}_{n},
$$

see Eq. (22) as well, where the vector $\overline{\boldsymbol{M}}^{(\hat{E})} \in \mathbb{R}^{n_{\mathrm{p}}}$ extracts the required nodal reaction forces. All the data of the simulation are in vector $\boldsymbol{s} \in \mathbb{R}^{n_{\mathrm{D}}}$.

The interpolation in time has to be applied to the force data of the testing machine and the FEM reaction force data (of a subset of nodes and directions) as well, see [19] for the computation of reaction forces in finite elements. Accordingly, we have, for example, $\boldsymbol{d}_{n}^{(\hat{E}) T}=\left\{F_{\exp n}^{(\hat{E})}, \tilde{\boldsymbol{u}}_{n}^{(\hat{E}) T}\right\}$ at time $t_{n}$ if the surface displacements are considered. This is the most common approach in comparing DIC and FEM. The comparison of displacements is in some situations very difficult, particularly when rigid body motions in the experiment superimpose the deformation caused by the movement of the testing machine's sample holders (which is the common case). This is very critical in small strain applications, where the resolution of DIC becomes sensitive. We make use of the option that rigid body motions are subtracted from the data. Alternatively, a strain measure can be chosen since it does not contain rigid body motions (but is influenced by comparing strains of DIC and FEM at different spatial positions). In the following investigations, we will thus, for the sake of simplicity, assume that the evaluation times, which are interpolated first, and the spatial evaluation points are identical.

Let $\boldsymbol{d}$ be the total experimental data as discussed previously, which is compared to the finite element results $\boldsymbol{S}(\boldsymbol{\kappa})$ concerned. The finite element results depend on the material parameters $\boldsymbol{\kappa} \in \mathbb{R}^{n_{\kappa}}$, which are the Lame constants $\boldsymbol{\kappa}^{T}=\{\Lambda, \mu\}$ (or the Young's modulus and the Poisson's number $\{E, \nu\}$ ) in the case of linear, isotropic elasticity, and for the case of transverse isotropy, $\kappa_{\mathrm{SP}}, \kappa_{\mathrm{C}}$, or $\kappa_{\mathrm{S}}$, see last paragraph in Sect. 2.1.

In a least-square sense, the square of the residual $\boldsymbol{r}(\kappa)=\boldsymbol{s}(\kappa)-\boldsymbol{d}$ or a weighted residual $\tilde{\boldsymbol{r}}(\boldsymbol{\kappa})=\boldsymbol{W r}(\boldsymbol{\kappa})=$ $\boldsymbol{W}\{\boldsymbol{s}(\boldsymbol{\kappa})-\boldsymbol{d}\}$ has to be minimized. According to [12], we weight the data by the number of data points and by an expected order of magnitude of a parameter, where $\boldsymbol{W} \in \mathbb{R}^{n_{\mathrm{D}} \times n_{\mathrm{D}}}$ is a diagonal matrix

$$
\boldsymbol{W}=\left[\begin{array}{ccc}
\boldsymbol{W}^{(1)} & & \\
& \ddots & \\
& & \boldsymbol{W}^{\left(n_{\exp }\right)}
\end{array}\right] \text { with } \boldsymbol{W}^{(\hat{E})}=\left[\begin{array}{lll}
\boldsymbol{W}_{0}^{(\hat{E})} & & \\
& \ddots & \\
& & \boldsymbol{W}_{n_{\mathrm{N}}^{(\hat{E})}}^{(\hat{E})}
\end{array}\right]
$$

If we take force data and displacement data into account, we have

$$
\boldsymbol{W}_{n}^{(\hat{E})}=\left[\begin{array}{ll}
w_{F}^{(\hat{E})} & \\
& w_{u}^{(\hat{E})} \boldsymbol{I}
\end{array}\right],
$$

where $w_{F}^{(\hat{E})}$ weights the force data

$$
w_{F}^{(\hat{E})}=\frac{1}{\max _{n=1, \ldots, n_{\mathrm{N}}^{(\hat{E})}}\left|F_{n}^{(\hat{E})}\right|}
$$


and $w_{u}^{(\hat{E})}$ are the weighting factors for the displacement data (or strain data)

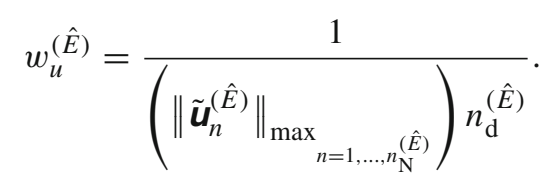

In the least-square sense, we have to minimize

$$
f(\kappa)=\frac{1}{2}\{\boldsymbol{W r}(\kappa)\}^{T} \boldsymbol{W r}(\kappa)=\frac{1}{2}\{\boldsymbol{W}\{\boldsymbol{s}(\boldsymbol{\kappa})-\boldsymbol{d}\}\}^{T}\{\boldsymbol{W}\{\boldsymbol{s}(\kappa)-\boldsymbol{d}\}\} \rightarrow \text { min. }
$$

There might be situations in which we have constraints (equality and inequality constraints) for the material parameters,

$$
\begin{aligned}
\boldsymbol{c}_{I}(\boldsymbol{\kappa}) & \leq \boldsymbol{0}, & \boldsymbol{c}_{I} \in \mathbb{R}^{m_{I}} \\
\boldsymbol{c}_{E}(\boldsymbol{\kappa}) & =\mathbf{0}, & \boldsymbol{c}_{E} \in \mathbb{R}^{m_{E}},
\end{aligned}
$$

where $m_{I}$ defines the number of inequality constraints and $m_{E}$ the number of equality constraints. Here, $m_{E}<n_{\kappa}$ should hold so that we do not obtain a system of nonlinear equations (or linear-depending on the problem). In the following theoretical investigations of identifiability, we assume for the sake of simplicity that there are no constraint conditions (18) in the following theoretical considerations.

A necessary condition for a minimum at $\kappa=\kappa^{*}$ requires a vanishing derivative (find the root of a nonlinear system)

$$
\boldsymbol{F}\left(\kappa^{*}\right)=\left.\frac{\mathrm{d} f}{\mathrm{~d} \boldsymbol{\kappa}}\right|_{\kappa=\kappa^{*}}=\boldsymbol{D}^{T}\left(\boldsymbol{\kappa}^{*}\right) \boldsymbol{W}^{T} \boldsymbol{W}\left\{\boldsymbol{s}\left(\boldsymbol{\kappa}^{*}\right)-\boldsymbol{d}\right\}=\mathbf{0},
$$

with the Jacobian (sensitivity matrix)

$$
\boldsymbol{D}(\boldsymbol{\kappa})=\frac{\mathrm{d} \tilde{r}(\kappa)}{\mathrm{d} \kappa}=\frac{\mathrm{d} \boldsymbol{s}(\kappa)}{\mathrm{d} \kappa}
$$

$\boldsymbol{D} \in \mathbb{R}^{n_{\mathrm{D}} \times n_{\kappa}}$, see, for example, [29,34,40]. Gauss-Newton-like methods require these derivatives. Since $\boldsymbol{s}_{n}^{(\hat{E}) T}(\boldsymbol{\kappa})=\left\{F_{\mathrm{FEM} n}^{(\hat{E})}(\boldsymbol{\kappa}) \tilde{\boldsymbol{u}}_{n}^{(\hat{E}) T}(\boldsymbol{\kappa})\right\}$ is looked for, we need the sensitivities of the resulting forces and the necessary displacements of the finite element simulation

$$
\frac{\mathrm{d} F_{\mathrm{FEM} n}^{(\hat{E})}(\boldsymbol{\kappa})}{\mathrm{d} \boldsymbol{\kappa}}=\overline{\boldsymbol{M}}^{(\hat{E}) T} \frac{\mathrm{d} \boldsymbol{p}_{n}^{(\hat{E})}(\boldsymbol{\kappa})}{\mathrm{d} \boldsymbol{\kappa}}, \quad \frac{\mathrm{d} \tilde{\boldsymbol{u}}_{n}^{(\hat{E})}(\boldsymbol{\kappa})}{\mathrm{d} \boldsymbol{\kappa}}=\tilde{\boldsymbol{M}}^{(\hat{E})} \frac{\mathrm{d} \boldsymbol{u}_{n}^{(\hat{E})}(\boldsymbol{\kappa})}{\mathrm{d} \boldsymbol{\kappa}},
$$

see Eq. (12) as well. The matrix $\tilde{\boldsymbol{M}}^{(\hat{E})} \in \mathbb{R}^{n_{\mathrm{d}}^{(\hat{E})} \times n_{\mathrm{u}}^{(\hat{E})}}$ extracts the required nodal displacements, which are compared to the experimental data (more precisely, which are used in the interpolation concept).

We follow two possibilities to determine the sensitivity matrix $\boldsymbol{D}$. First, there is a purely analytical approach to provide the sensitivity matrix, which we call internal numerical differentiation (IND), see [40]. The external numerical differentiation (END) provides the sensitivity matrix by numerical differentiation.

In the case of IND (applied to finite element models), we treat the matrix representation of finite elements

$$
\boldsymbol{G}(\boldsymbol{u}(\kappa), \boldsymbol{p}(\kappa), \kappa)=\left\{\begin{array}{c}
\boldsymbol{g}(\boldsymbol{u}(\kappa), \boldsymbol{\kappa}) \\
\boldsymbol{g}(\boldsymbol{u}(\kappa), \kappa)-\boldsymbol{p}(\kappa)
\end{array}\right\}=0 .
$$

The indices $(\hat{E})$ and $n$ are omitted for the sake of brevity. The first set of equations defines the discretized principle of virtual displacements, and the second set of equations defines the resulting reaction forces at those degrees of freedom, where displacements are prescribed. In the case of linear elasticity, these equations read

$$
\begin{aligned}
& \boldsymbol{g}(\boldsymbol{u}(\kappa), \boldsymbol{\kappa})=\boldsymbol{K}(\kappa) \boldsymbol{u}(\kappa)+\overline{\boldsymbol{K}}(\kappa) \overline{\boldsymbol{u}}-\overline{\boldsymbol{p}}, \\
& \overline{\boldsymbol{g}}(\boldsymbol{u}(\kappa), \kappa)=\overline{\boldsymbol{K}}^{T}(\kappa) \boldsymbol{u}(\kappa)+\overline{\overline{\boldsymbol{K}}}(\kappa) \overline{\boldsymbol{u}} .
\end{aligned}
$$

$\overline{\boldsymbol{p}} \in \mathbb{R}^{n_{\mathrm{u}}}$ are prescribed equivalent nodal forces, and $\boldsymbol{p} \in \mathbb{R}^{n_{\mathrm{p}}}$ are the unknown nodal reaction forces. $\boldsymbol{u} \in \mathbb{R}^{n_{\mathrm{u}}}$ are the unknown and $\overline{\boldsymbol{u}} \in \mathbb{R}^{n_{\mathrm{p}}}$ the prescribed nodal displacements. Alternatively, we can write

$$
\boldsymbol{g}(t, \boldsymbol{u}(\boldsymbol{\kappa}), \boldsymbol{\kappa})=\sum_{e=1}^{n_{\mathrm{el}}} \boldsymbol{Z}^{e T}\left\{\sum_{j=1}^{n_{\mathrm{GP}}^{e}} w^{e(j)} \mathbf{B}^{e(j)} \mathbf{C}^{e(j)} \mathbf{E}^{e(j)} \operatorname{det} \mathbf{J}^{e(j)}\right\}-\overline{\boldsymbol{p}}(t),
$$




$$
\overline{\boldsymbol{g}}(t, \boldsymbol{u}(\boldsymbol{\kappa}), \boldsymbol{\kappa})=\sum_{e=1}^{n_{\mathrm{el}}} \overline{\boldsymbol{Z}}^{e T}\left\{\sum_{j=1}^{n_{\mathrm{GP}}^{e}} w^{e(j)} \mathbf{B}^{e(j)} \mathbf{C}^{e(j)} \mathbf{E}^{e(j)} \operatorname{det} \mathbf{J}^{e(j)}\right\} .
$$

Although the material parameters might be linearly embedded in the expressions (25) and (26), the resulting identification scheme does not lead to a linear least-square method, because the solutions $\mathrm{d} \boldsymbol{u}(\boldsymbol{\kappa}) / \mathrm{d} \boldsymbol{\kappa}$ and $\mathrm{d} \boldsymbol{p} / \mathrm{d} \boldsymbol{\kappa}$ are only implicitly given (by the solutions of a linear system). $\boldsymbol{K}=\boldsymbol{K}^{T} \in \mathbb{R}^{n_{\mathrm{u}} \times n_{\mathrm{u}}}$ defines the classical symmetric stiffness matrix, and $\overline{\boldsymbol{K}} \in \mathbb{R}^{n_{\mathrm{u}} \times n_{\mathrm{p}}}$ and $\overline{\overline{\boldsymbol{K}}} \in \mathbb{R}^{n_{\mathrm{p}} \times n_{\mathrm{p}}}$ are the remaining stiffness matrices:

$$
\begin{aligned}
& \boldsymbol{K}=\sum_{e=1}^{n_{\mathrm{el}}} \boldsymbol{Z}^{e T}\left[\sum_{j=1}^{n_{\mathrm{GP}}^{e}} w^{e(j)} \mathbf{B}^{e(j)} \mathbf{C}^{e(j)} \mathbf{B}^{e(j)} \operatorname{det} \mathbf{J}^{e(j)}\right] \boldsymbol{Z}^{e} \\
& \overline{\boldsymbol{K}}=\sum_{e=1}^{n_{\mathrm{el}}} \overline{\boldsymbol{Z}}^{e T}\left[\sum_{j=1}^{n_{\mathrm{GP}}^{e}} w^{e(j)} \mathbf{B}^{e(j)} \mathbf{C}^{e(j)} \mathbf{B}^{e(j)} \operatorname{det} \mathbf{J}^{e(j)}\right] \boldsymbol{Z}^{e} \\
& \overline{\overline{\boldsymbol{K}}}=\sum_{e=1}^{n_{\mathrm{el}}} \overline{\boldsymbol{Z}}^{e T}\left[\sum_{j=1}^{n_{\mathrm{GP}}^{e}} w^{e(j)} \mathbf{B}^{e(j)} \mathbf{C}^{e(j)} \mathbf{B}^{e(j)} \operatorname{det} \mathbf{J}^{e(j)}\right] \overline{\boldsymbol{Z}}^{e}
\end{aligned}
$$

$\boldsymbol{Z}^{e} \in \mathbb{R}^{n_{\mathrm{u}}^{e} \times n_{\mathrm{u}}}$ and $\overline{\boldsymbol{Z}}^{e} \in \mathbb{R}^{n_{\mathrm{u}}^{e} \times n_{\mathrm{p}}}$ symbolize the assembling matrices, which are not programmed but defined in order to describe the assembling procedure (for the notation used, see [19]. $n_{\mathrm{u}}^{e}$ is the number of element nodal displacement degrees of freedom, $w^{e(j)}$ are the weighting factors of the Gauss integration in an element, $n_{\mathrm{GP}}^{e}$ symbolize the number of Gauss points within one element, and $\mathbf{B}^{e(j)} \in \mathbb{R}^{6 \times n_{\mathrm{u}}^{e}}$ defines the strain-displacement matrix of element $e$ evaluated at the $j$ th Gauss point $\xi^{(j)} \in \mathbb{R}^{3}, j=1, \ldots, n_{\mathrm{GP}}^{e}$,

$$
\mathbf{E}^{e(j)}=\mathbf{B}^{e(j)} \mathbf{u}^{e}=\mathbf{B}^{e(j)}\left\{\boldsymbol{Z}^{e} \boldsymbol{u}+\overline{\boldsymbol{Z}}^{e} \overline{\mathbf{u}}(t)\right\}, \quad \mathbf{E}^{e(j)} \in \mathbb{R}^{6}
$$

with the element nodal displacements $\mathbf{u}^{e} \in \mathbb{R}^{n_{\mathrm{u}}^{e}}$. The prescribed nodal displacements $\overline{\boldsymbol{u}}(t)$ depend explicitly on the time $t$, i.e., the load step in the case of elasticity. $\mathbf{J}^{e(j)} \in \mathbb{R}^{3 \times 3}$ defines the Jacobi matrix of the coordinate transformation between reference element coordinates and global coordinates. In order to obtain the sensitivities (20), the chain rule is applied to Eq. (22) leading to the linear system

$$
\frac{\partial \boldsymbol{g}}{\partial \boldsymbol{u}} \frac{\mathrm{d} \boldsymbol{U}}{\mathrm{d} \boldsymbol{\kappa}}+\frac{\partial \boldsymbol{g}}{\partial \boldsymbol{\kappa}}=\boldsymbol{O} \Rightarrow \boldsymbol{K} \frac{\mathrm{d} \boldsymbol{u}}{\mathrm{d} \boldsymbol{\kappa}}=-\frac{\partial \boldsymbol{g}}{\partial \boldsymbol{\kappa}} \rightsquigarrow \frac{\mathrm{d} \boldsymbol{u}}{\mathrm{d} \boldsymbol{\kappa}}
$$

and the resulting sensitivities

$$
\frac{\mathrm{d} \boldsymbol{p}}{\mathrm{d} \boldsymbol{\kappa}}=\frac{\partial \overline{\boldsymbol{g}}}{\partial \boldsymbol{u}} \frac{\mathrm{d} \boldsymbol{u}}{\mathrm{d} \boldsymbol{\kappa}}+\frac{\partial \overline{\boldsymbol{g}}}{\partial \boldsymbol{\kappa}} \Rightarrow \frac{\mathrm{d} \boldsymbol{p}}{\mathrm{d} \boldsymbol{\kappa}}=\overline{\boldsymbol{K}} \frac{\mathrm{d} \boldsymbol{u}}{\mathrm{d} \boldsymbol{\kappa}}+\frac{\partial \overline{\boldsymbol{g}}}{\partial \boldsymbol{\kappa}} .
$$

Obviously, after an LU decomposition of the stiffness matrix $\boldsymbol{K}$ in Eq. (31), $n_{\kappa}$ additional back-substitutions are required to obtain the derivative $\mathrm{d} \boldsymbol{u} / \mathrm{d} \boldsymbol{\kappa}$ (the superscript $(\hat{E})$ is omitted for brevity). Afterward, the sensitivities of the reaction force are only given by matrix-matrix products, see Eq. (32).

Alternatively to IND, it is possible to apply END, which means performing numerical differentiation to obtain the sensitivities. In this case, the derivatives are obtained by

$$
\frac{\mathrm{d} \boldsymbol{u}_{n}^{(\hat{E})}}{\mathrm{d} \boldsymbol{\kappa}} \approx \sum_{j=1}^{n_{\kappa}} \frac{\boldsymbol{u}_{n}^{(\hat{E})}\left(\boldsymbol{\kappa}+\Delta \kappa_{j} \overline{\boldsymbol{e}}_{j}\right)-\boldsymbol{u}_{n}^{(\hat{E})}(\boldsymbol{\kappa})}{\Delta \kappa_{j}} \overline{\boldsymbol{e}}_{j}^{T}=\sum_{i=1}^{n_{\mathrm{u}}^{(\hat{E})}} \sum_{j=1}^{n_{\kappa}} \frac{u_{n i}^{(\hat{E})}\left(\boldsymbol{\kappa}+\Delta \kappa_{j} \overline{\boldsymbol{e}}_{j}\right)-u_{n i}^{(\hat{E})}(\boldsymbol{\kappa})}{\Delta \kappa_{j}} \boldsymbol{e}_{i} \overline{\boldsymbol{e}}_{j}^{T}
$$

with the "unit vectors" $\boldsymbol{e}_{i} \in \mathbb{R}^{n_{\mathrm{u}}^{(\hat{E})}}$ (all entries are zero except for one having a 1 in row $i$ ) and $\overline{\boldsymbol{e}}_{j} \in \mathbb{R}^{n_{\kappa}}$. Furthermore, we have

$$
\frac{\mathrm{d} \boldsymbol{p}_{n}^{(\hat{E})}}{\mathrm{d} \boldsymbol{\kappa}} \approx \sum_{j=1}^{n_{\kappa}} \frac{\boldsymbol{p}_{n}^{(\hat{E})}\left(\boldsymbol{\kappa}+\Delta \kappa_{j} \overline{\boldsymbol{e}}_{j}\right)-\boldsymbol{p}_{n}^{(\hat{E})}(\boldsymbol{\kappa})}{\Delta \kappa_{j}} \overline{\boldsymbol{e}}_{j}^{T}=\sum_{i=1}^{n_{\mathrm{p}}^{(\hat{E})}} \sum_{j=1}^{n_{\kappa}} \frac{p_{n i}^{(\hat{E})}\left(\boldsymbol{\kappa}+\Delta \kappa_{j} \overline{\boldsymbol{e}}_{j}\right)-p_{n i}^{(\hat{E})}(\boldsymbol{\kappa})}{\Delta \kappa_{j}} \overline{\overline{\boldsymbol{e}}}_{i} \overline{\boldsymbol{e}}_{j}^{T}
$$


(here, of course, $\overline{\overline{\boldsymbol{e}}}_{i} \in \mathbb{R}^{n_{\mathrm{p}}^{(\hat{E})}}$ holds). In this case, the entire finite element program has to be called $n_{\kappa}+1$ times. Although END is computationally not as efficient as IND, it can be applied to commercial (black box) programs. The increments $\Delta \kappa_{j}$ must be adapted to the accuracies of the finite element program (single or double precision — not only for the internal numerical computations but also for the input and output reads and writes).

There are further questions that should be treated in the parameter identification. Since the applied optimizers will-more or less-provide a solution, we have to ask the question about the quality of the material parameters found. As discussed in Beck and Arnold [3], the confidence interval determination and the identifiability concept can be applied. For an application of these concepts in solid mechanics, see [14,20]. A common indicator of the identification quality is given by the value $R^{2}=1-\left(\sum_{i=1}^{n_{\mathrm{d}}}\left(d_{i}-s_{i}\right)^{2}\right) /\left(\sum_{i=1}^{n_{\mathrm{d}}}\left(d_{i}-\bar{d}\right)^{2}\right)$ with $\bar{d}=\left(1 / n_{\mathrm{d}}\right) \sum_{i=1}^{n_{\mathrm{d}}} d_{i}, R^{2} \leq 1$. If $R^{2} \approx 1$, one obtains a very good fit. However, this does not say anything about the quality of the material parameters itself. Thus, we are interested in further measures that could indicate the identification quality. As described in Eq. (17) a necessary condition for the minimum problem is given by Eq. (19) if we assume no constraint conditions. $\boldsymbol{\kappa}^{*}$ is a solution computed by the optimization program. Since there is a lot of knowledge in linear least-square problems, we follow the subsequent idea. We approximate the objective function (17) in the local minimum $\kappa^{*}$ by a quadratic function,

$$
\hat{f}(\kappa)=f\left(\kappa^{*}\right)+\left.\left\{\frac{\mathrm{d} f(\boldsymbol{\kappa})}{\mathrm{d} \kappa}\right\}^{T}\right|_{\kappa=\kappa^{*}} \Delta \kappa+\left.\frac{1}{2} \Delta \kappa^{T}\left[\frac{\mathrm{d}^{2} f(\kappa)}{\mathrm{d} \kappa \mathrm{d} \kappa}\right]\right|_{\kappa=\kappa^{*}} \Delta \kappa,
$$

with $\Delta \kappa=\kappa-\kappa^{*}$. The third term contains the Hessian matrix

$$
\boldsymbol{H}(\boldsymbol{\kappa})=\frac{\mathrm{d}^{2} f(\boldsymbol{\kappa})}{\mathrm{d} \boldsymbol{\kappa} \mathrm{d} \boldsymbol{\kappa}}=\left[\frac{\partial^{2} f(\boldsymbol{\kappa})}{\partial \kappa_{i} \partial \kappa_{j}}\right]=\left[\sum_{k=1}^{n_{\mathrm{d}}} w_{k k}^{2}\left(\frac{\partial^{2} s_{k}(\boldsymbol{\kappa})}{\partial \kappa_{i} \partial \kappa_{j}}\left(s_{k}(\boldsymbol{\kappa})-d_{k}\right)+\frac{\partial s_{k}(\boldsymbol{\kappa})}{\partial \kappa_{i}} \frac{\partial s_{k}(\boldsymbol{\kappa})}{\partial \kappa_{j}}\right)\right] .
$$

Subsequently, we make the assumption that

$$
\boldsymbol{H} \approx \boldsymbol{D}^{T} \boldsymbol{D}=\left[w_{k k}^{2} \frac{\partial s_{k}(\boldsymbol{\kappa})}{\partial \kappa_{i}} \frac{\partial s_{k}(\boldsymbol{\kappa})}{\partial \kappa_{j}}\right]
$$

holds, which is justified as long as $\|\boldsymbol{r}\|=\|\boldsymbol{s}-\boldsymbol{d}\|$ is small. Beveridge and Schechter [5], Beck and Arnold [3] proposed the concept of identifiability, which is related to the question whether there is an unique, local minimum. In the case of fulfilling the necessary condition (19) and for nonvanishing and positive definite sub-determinants of the Hessian,

$$
D_{r}=\left|\begin{array}{cccc}
H_{11} & H_{12} & \ldots & H_{1 r} \\
H_{21} & H_{22} & \ldots & H_{2 r} \\
\vdots & & & \vdots \\
H_{r 1} & H_{r 2} & \ldots & H_{r r}
\end{array}\right|,
$$

i.e., $D_{r}>0$ for $r=1, \ldots, n_{\kappa}$, a unique local minimum exists. The condition $\operatorname{det} \boldsymbol{H} \neq 0$ has to be checked so that we can state whether there might be a unique solution. Furthermore, it is known that the closer $D_{r}$ is to zero, the more sensitive the optimization result is to small perturbations. In other words, we look at the local convexity of the objective function. In the case of a "long valley," local uniqueness is not given, see for simple examples [14].

To our experience, it turned out that the identifiability investigation should be studied with "synthetic data," i.e., one solves the boundary value problem of the experiment by means of finite elements, with some "reasonable" parameters and tries to re-identify the parameters using the generated data assuming that these are experimental results. Then, investigating the Hessian indicates whether the parameters are addressed by the experiments, see $[14,20]$. If one uses experimental data directly, the influence of dispersions makes it very hard to interpret the results.

One additional measure for the quality of identification is the confidence interval. For this purpose, we need the covariance matrix

$$
\boldsymbol{P}=s^{2} \boldsymbol{H}^{-1}\left(\boldsymbol{\kappa}^{*}\right) \text {. }
$$

Here, we have the standard deviation

$$
s^{2}=\frac{1}{n_{\mathrm{D}}-1} \boldsymbol{r}^{T}\left(\boldsymbol{\kappa}^{*}\right) \boldsymbol{r}\left(\boldsymbol{\kappa}^{*}\right) .
$$




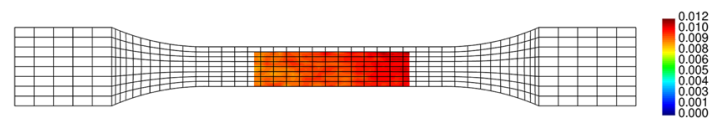

(a) Axial strains $\varepsilon_{11}$

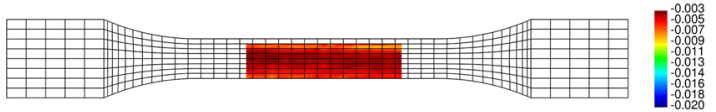

(b) Lateral strains $\varepsilon_{22}$

Fig. 7 Strain distribution of pure resin specimens

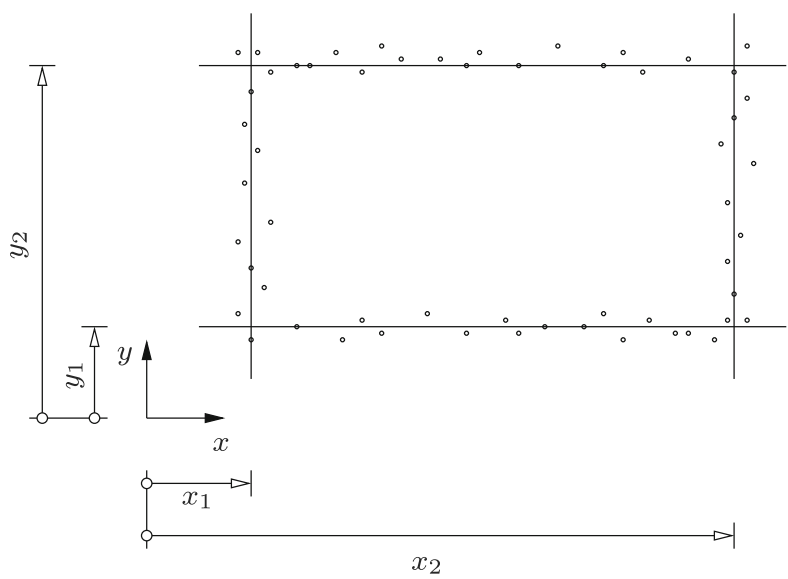

Fig. 8 Sketch of length and width determination on the basis of DIC images

The confidence interval is defined by

$$
\kappa_{\mathrm{conf}}=\kappa^{*} \pm \Delta \kappa,
$$

which is based on the diagonal components of the covariance matrix,

$$
\Delta \kappa_{i}=\sqrt{P_{i i}}, \quad i=1, \ldots, n_{\mathrm{d}}
$$

This is an indicator whether the material parameters are correlated, essentially depending on the experiments chosen. The correlation is provided by

$$
\boldsymbol{C}=\left[c_{i j}\right] \quad \text { with } \quad c_{i j}=\frac{P_{i j}}{\sqrt{P_{i i}} \sqrt{P_{j j}}}, \quad i, j=1, \ldots, n_{\kappa}
$$

The diagonal terms are $c_{i i}=1$ and the off-diagonal elements represent the linear correlation between the parameters $\kappa_{i}$ and $\kappa_{j},\left|c_{i j}\right| \leq 1$. The correlation matrix only makes sense if the Hessian is regular and the standard deviation is nonzero.

These pieces of information are chosen in the experiments described later on. Furthermore, we have to remark that we draw on the MATLAB tool lsqnonlin.m as an optimizer if there are no constraints assumed and on the in-house finite element program tasafem to solve the boundary value problem where the constitutive models are implemented. If there are constraint conditions, see (18), then we draw on the MATLAB routine fmincon.m.

\subsection{Pure resin}

First, we carry out five tensile tests on pure resin specimens and measure the axial and lateral strains $\varepsilon_{11}$ and $\varepsilon_{22}$, respectively-see Fig. 7, where the region and strain distribution is exemplarily shown. In the following, we are interested in comparing both the parameter identification on the basis of analytical modeling and the calibration applying the numerical results of the DIC/FEM approach. 


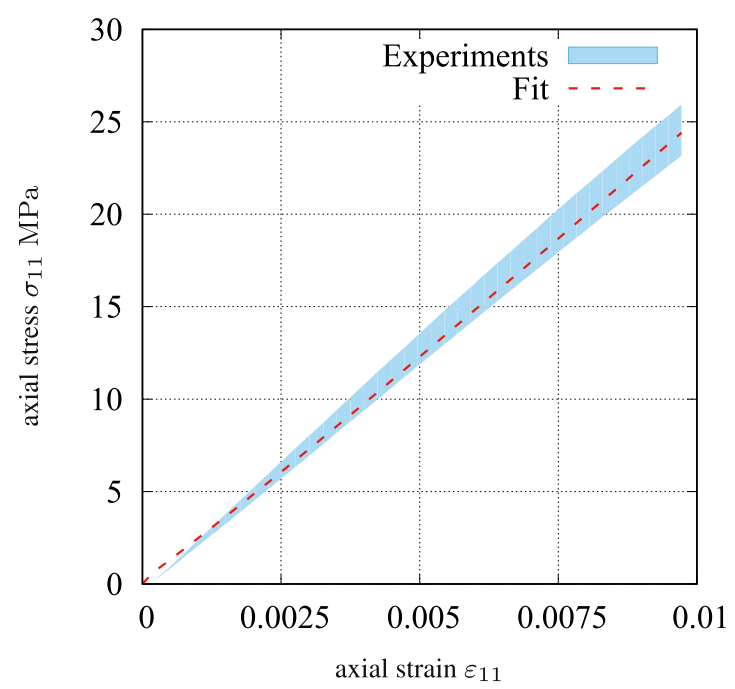

(a) Axial stress vs axial strain pure resin specimens

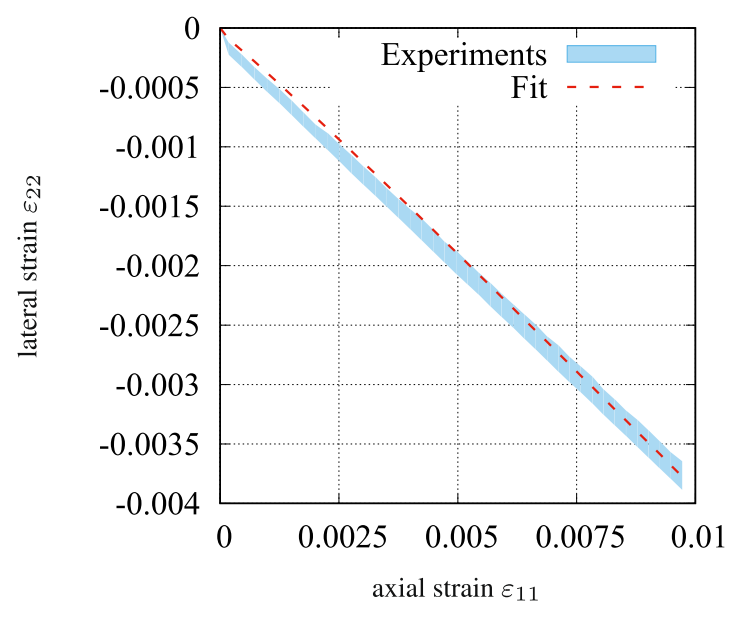

(b) Lateral strain vs axial strain for pure resin specimens

Fig. 9 Calibrated experiment-fit diagram

\subsubsection{Analytical approach (pure resin-isotropy)}

We define two lines by the mean values of the coordinates concerned in the DIC data, each in axial and lateral direction (in each experiment), and determine the original distances $L_{0}^{(\hat{E})}$ (length) and $B_{0}^{(\hat{E})}$ (width), see Fig. 8 , For this purposes, we assume that the coordinates of the DIC images at time $t_{n}$ are given. Then, we define vertical and horizontal lines representing the mean values of the $x$ and $y$ coordinates, which are $x_{1}, x_{2}, y_{1}$, and $y_{2}$-leading to $L=x_{2}-x_{1}$ and $B=y_{2}-y_{1}$. This is done for the reference image (reference configuration) and the current configurations. Then, for each loading step $L_{n}^{(\hat{E})}$ and $B_{n}^{(\hat{E})}, n=1, \ldots, n_{\mathrm{N}}^{(\hat{E})}, \hat{E}=1, \ldots, 5$, $n_{\exp }=5$, are known. Using the measured forces $F_{n}^{(\hat{E})}$, we obtain the axial stress $\sigma_{11}$, the axial strains $\varepsilon_{11}$, and the lateral strains $\varepsilon_{22}$ by

$$
\sigma_{11}^{(\hat{E})}=\frac{F_{n}^{(\hat{E})}}{A_{0}}, \quad \varepsilon_{11}^{(\hat{E})}=\frac{L_{n}^{(\hat{E})}-L_{0}^{(\hat{E})}}{L_{0}^{(\hat{E})}}, \quad \varepsilon_{22}(\hat{E})=\frac{B_{n}^{(\hat{E})}-B_{0}^{(\hat{E})}}{B_{0}^{(\hat{E})}} .
$$

Since there are small deviations in the specimens' geometries, we take a mean value for the cross section $A_{0}=$ $\left(\sum_{\hat{E}=1}^{n_{\exp }} A_{0}^{(\hat{E})}\right) / n_{\exp }$ in Eq. (44) 1 to determine the axial stresses, see Fig. 3 a for the force/displacement behavior. These data serve to apply a least-square scheme to determine the Young's modulus $E=2510 \pm 196 \mathrm{MPa}$ and the Poisson's number $v=0.39 \pm 0.03$. The result of the identification is shown in Fig. 9.

We are also interested in determining the other set of parameters $\Lambda(E, v)$ and $\mu(E, v)$, see relation (9). To obtain the uncertainty from that representation, we draw on the linear error propagation theory, see [45]. A function $f(\kappa)$ with the estimated deviation $\Delta \kappa$ yields the uncertainty

$$
\delta f=\sqrt{\sum_{k=1}^{n_{\kappa}}\left(\frac{\partial f}{\partial \kappa_{k}} \Delta \kappa_{k}\right)^{2}}
$$

i.e., $f \pm \delta f$, evaluated at the best fit $\boldsymbol{\kappa}$ obtained by the other set of parameters (for the best fit, we assume the least-square result). Here, we take $\Delta \kappa_{k}$ as the standard deviation. Then, we obtain

$$
\Lambda=3201 \pm 1079 \mathrm{MPa}, \quad \text { and } \mu=903 \pm 73 \mathrm{MPa}
$$

for $f=\Lambda$ and $f=\mu$ respectively. Particularly, a small change in the Poisson's number $v$ yields a big change in $\Lambda$. 


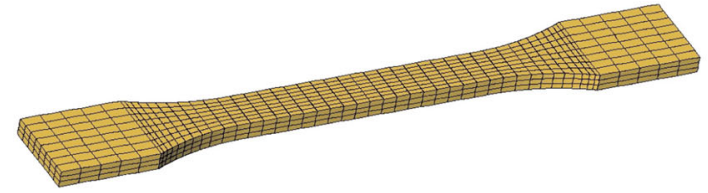

(a) Dog-bone specimen for pure resin

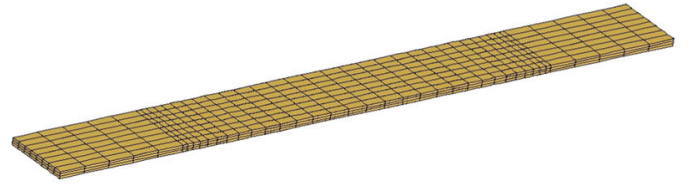

(b) Flat specimen for reinforced composite

Fig. 10 Finite element meshes using 20-noded hexahedral elements

\subsubsection{Finite elements and DIC (pure resin-isotropy)}

In the following, we perform the parameter identification procedure of Sect. 3.1 using the DIC data of the pure matrix material to determine the material parameters $(E, \nu)$, and indirectly $\kappa=\{\Lambda, \mu\}$ as discussed in the previous Sect. 3.2.1. We draw on the mesh in Fig. 10a. The DIC data are given by five tests, each with 40 load steps. Frequently, the displacement data are used to perform the parameter identification process. It turned out that this has substantial drawbacks regarding the results of the parameters since there are always rigid body motions in the experimental data. Thus, we draw on the surface strains calculated for both, DIC data, see, for example, Fig. 7, and the finite element mesh according to [16]. In both cases, the motion of the points (in the context of finite elements, the surface nodes) are interpolated using a triangulation. The in-plane strains are determined in each triangle, or the two principal strains are calculated (maximum and minimum strain). At the nodes of the finite element simulation, we determine the mean value of the triangle results directly connected to the node. Then, the FEM values are projected onto the DIC triangles and compared. This leads to a huge amount of spatial data points (approximately 22,000 data points per test) and only few data for the external forces (40 data points per test). The least-square problem, weighted as explained in Sec. 3.1, leads to $E=2453 \pm 34 \mathrm{MPa}$ and $v=0.39 \pm 0.007$. If we draw on Eq. (45), the resulting Lame constants are $\Lambda=3128 \pm 243 \mathrm{MPa}$ and $\mu=882 \pm 13 \mathrm{MPa}$, where the "confidence interval" is smaller than for the analytical approach. Furthermore, the identified values are very close to the values identified with the analytical considerations. Thus, the DIC/FEM approach yields similar results, and the parameters are considered reliable.

Since we apply both sensitivities with fully analytical derivatives (IND) and classical functional matrices based on numerical differentiation (END), we can compare the computational time. In the case of IND, the computational time required is around $189 \mathrm{~s}$ while END takes around $711 \mathrm{~s}$. This is a factor of 3.8, or, roughly estimated, IND requires only a quarter of the computing time compared to END. The computations are done using the in-house finite element program TASAFEM on a four-core computer with an Intel(R) Core(TM) i74770 processor (8MB Cache, $3.4 \mathrm{GHz}$ ) and 16GB memory. Furthermore, we apply the linear solver PARDISO, [39], addressing 8 threads.

\subsection{Fiber reinforcement}

In the case of transverse isotropy, we are not aware of publications regarding material parameter identification that treat the whole set of five parameters in transverse isotropy, except for the discussion in Christensen [8]. Commonly, plane stress conditions are applied requiring only four material parameters-see discussion in Christensen [8] as well-if the thickness change is not of interest. If computations with three-dimensional continuum finite elements are performed, all material parameters are required. There are three simple tests that can be treated, namely

- simple tension of a $\gamma=0^{\circ}$ specimen, where the axial and lateral strains are measured,

- simple tension of a $\gamma=90^{\circ}$ specimen, where the axial and lateral strains are measured (here, only the axial information is necessary),

- and a shear test (fiber orientation parallel to loading direction).

Using these experiments, only four material parameters can be addressed. One additional equi-biaxial testpresented in Christensen [8] — where a constant layer thickness is assumed, is commonly not applicable, but would finalize the material parameter identification procedure. Here, we propose a compression test that can be carried out in a uniaxial compression testing machine, see Sect. 2.2.3. Of course, the latter test provides only force and displacement data and no images by a DIC system, i.e., the data have an integral character. It will be shown that the sensitive compression test data might lead to problems in a reliable determination of the parameters so that further investigations have to be performed. 
We will also show that the DIC/FEM approach is not able to overcome the problem without the compression test, which is commonly assumed by applying in-plane information with various fiber angles $\gamma$. A reliable "compression" test is essential to obtain reliable material parameters.

First, we recap the analytical approach using the assumption of homogeneous deformations. Second, we generate "synthetic data" to show that non-identifiability is apparent for the "DIC"/FEM approach if we choose $0^{\circ}, 45^{\circ}, 90^{\circ}$, and shear data. Identifiability is given only for $0^{\circ}, 90^{\circ}$, shear, and compression tests. Afterward, real DIC data are taken into account to identify the material parameters, which are compared to the analytical approach, i.e., where we assume homogeneity. Supplementary information about the internal and external numerical differentiation is given as well.

\subsubsection{Analytical parameter identification (transversal isotropy)}

Under the assumption of homogeneous deformations, we are able to determine uniquely the five material parameters. According to Christensen [8], we need four tests.

1. First, a tension test for specimens with $\mathbf{a}=\mathbf{e}_{1}$ in $\mathbf{e}_{1}$-direction. We prescribe $\varepsilon_{11}$ and measure both $\sigma_{11}$ by the testing machine and the lateral strains $\varepsilon_{22}$ by the DIC system, see the scheme in Appendix 3.2.1. Here, we draw on the representation of the compliance matrix (74) with the stress and strain state $\mathbf{T}^{T}=$ $\left\{\sigma_{11}, 0,0,0,0,0\right\}$ and $\mathbf{E}^{T}=\left\{\varepsilon_{11}, \varepsilon_{22}, \varepsilon_{33}, 0,0,0\right\}$. There, we obtain from the first equation of $\mathbf{E}=\mathbf{S}_{0} \circ \mathbf{T}$

$$
\begin{aligned}
& \varepsilon_{11}=\frac{\sigma_{11}}{E_{11}} \\
& \varepsilon_{22}=-\frac{\nu_{12}}{E_{11}} \sigma_{11}=-v_{12} \varepsilon_{11} \quad \Longrightarrow \quad v_{12}=-\frac{\varepsilon_{22}}{\varepsilon_{11}}
\end{aligned}
$$

2. Second, a tensile test in $\mathbf{e}_{1}$-direction but with fiber orientation $\mathbf{a}=\mathbf{e}_{2}$ can be performed. There are two possibilities of evaluating the resulting equations. Either, the compliance matrix is provided in a rotated form, $\mathbf{E}=\mathbf{S}_{90^{\circ}} \mathbf{T}$, or we take the compliance matrix (74), i.e., $\mathbf{E}=\mathbf{S}_{0} \circ \mathbf{T}$, but applying a stress $\sigma_{22}$ in $\mathbf{e}_{2}$-direction. This yields

$$
\begin{aligned}
& \varepsilon_{22}=\frac{\sigma_{22}}{E_{22}} \\
& \varepsilon_{11}=-\frac{\nu_{21}}{E_{22}} \sigma_{22}=-v_{21} \varepsilon_{22} \quad \Longrightarrow \quad v_{21}=-\frac{\varepsilon_{11}}{\varepsilon_{22}}
\end{aligned}
$$

The last equation does not provide any additional information, since

$$
\frac{v_{12}}{E_{11}}=\frac{v_{21}}{E_{22}}
$$

holds (symmetry of the compliance matrix). From the two tensile tests, we thus only obtain three material parameters, $E_{11}, E_{22}$ and $v_{12}$ (and, accordingly, $v_{21}$ ). If we transfer this to a specimen with $\mathbf{a}=\mathbf{e}_{2}$ and a loading direction $\mathbf{e}_{1}$, the Young's modulus $E_{22}$ is obtained by

$$
E_{22}=\frac{\sigma_{11}}{\varepsilon_{11}}
$$

since $\sigma_{11}$ and $\varepsilon_{11}$ represent the measured axial information.

3. The third test is the shear test of Sect. 2.2.2. Here, we obtain the shear modulus

$$
\gamma_{12}=\frac{1}{\mu_{12}} \tau_{12} \quad \Longrightarrow \mu_{12}=\frac{\tau_{12}}{\gamma_{12}}
$$

from the fourth equation in $\mathbf{E}=\mathbf{S}_{0^{\circ}} \mathbf{T}$.

4. The final test is given by the compression test yielding the stress and strain state $\mathbf{T}^{T}=\left\{\sigma_{11}, \sigma_{22}, \sigma_{33}, 0,0,0\right\}$ and $\mathbf{E}^{T}=\left\{0,0, \varepsilon_{33}, 0,0,0\right\} . \varepsilon_{33}$ is prescribed, and $\sigma_{33}$ is measured using the compression testing machine. In lateral direction, we measure the stresses $\sigma_{22}$ and $\sigma_{33}$. Here, we choose $\mathbf{a}=\mathbf{e}_{1}$. Using the first equation in 
Table 1 Identified parameters $\kappa_{\mathrm{S}}$ and estimated parameters $\kappa_{\mathrm{SP}}$ and $\boldsymbol{\kappa}_{\mathrm{C}}$ based on the assumption of homogeneous deformations (for the 2nd column, $v_{32}$ is not identified, but calculated by Eq. (59))

\begin{tabular}{|c|c|c|c|c|}
\hline Parameter & Dimension & Analytical approach & Christensen formula & Synthetic compression test \\
\hline \multicolumn{5}{|l|}{$\kappa_{\mathrm{S}}$} \\
\hline$E_{11}$ & $\mathrm{~N} \mathrm{~mm}^{-2}$ & $44,777 \pm 60$ & $44,777 \pm 60$ & $44,777 \pm 60$ \\
\hline$E_{22}$ & $\mathrm{~N} \mathrm{~mm}^{-2}$ & $12,964 \pm 59$ & $12,964 \pm 59$ & $12,964 \pm 59$ \\
\hline$v_{12}$ & - & $0.30 \pm 0.07$ & $0.30 \pm 0.07$ & $0.30 \pm 0.07$ \\
\hline$\mu_{12}$ & $\mathrm{~N} \mathrm{~mm}^{-2}$ & $3385 \pm 173$ & $3385 \pm 173$ & $3385 \pm 173$ \\
\hline$v_{32}$ & - & $0.02 \pm 0.0006$ & 0.39 & 0.35 \\
\hline \multicolumn{5}{|c|}{ 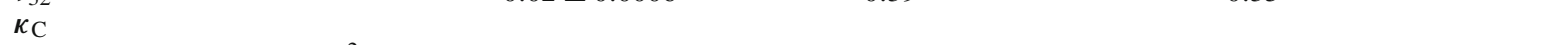 } \\
\hline$C_{11}$ & $\mathrm{~N} \mathrm{~mm}^{-2}$ & $47,900 \pm 3195$ & $50,016 \pm 5750$ & $49,637 \pm 5150$ \\
\hline$C_{22}$ & $\mathrm{~N} \mathrm{~mm}^{-2}$ & $13,435 \pm 477$ & $16,571 \pm 2892$ & $15,856 \pm 1149$ \\
\hline$C_{12}$ & $\mathrm{~N} \mathrm{~mm}^{-2}$ & $4710 \pm 2565$ & $7900 \pm 5074$ & $7330 \pm 4264$ \\
\hline$C_{23}$ & $\mathrm{~N} \mathrm{~mm}^{-2}$ & $774 \pm 474$ & $7260 \pm 3736$ & $6253 \pm 1147$ \\
\hline$C_{66}$ & $\mathrm{~N} \mathrm{~mm}^{-2}$ & $3385 \pm 173$ & $3385 \pm 173$ & $3385 \pm 173$ \\
\hline \multicolumn{5}{|l|}{$\kappa_{\mathrm{SP}}$} \\
\hline$\alpha$ & $\mathrm{N} \mathrm{mm}^{-2}$ & $3936 \pm 2092$ & $640 \pm 3642$ & $1076 \pm 3117$ \\
\hline$\beta$ & $\mathrm{N} \mathrm{mm}^{-2}$ & $38,375 \pm 1622$ & $37,246 \pm 2496$ & $37,295 \pm 2337$ \\
\hline$\mu_{T}$ & $\mathrm{~N} \mathrm{~mm}^{-2}$ & $6330 \pm 29$ & $4655 \pm 462$ & $4802 \pm 22$ \\
\hline$\Lambda$ & $\mathrm{N} \mathrm{mm}^{-2}$ & $774 \pm 474$ & $7260 \pm 3736$ & $6253 \pm 1147$ \\
\hline$\mu_{L}$ & $\mathrm{~N} \mathrm{~mm}^{-2}$ & $3385 \pm 173$ & $3385 \pm 173$ & $3385 \pm 173$ \\
\hline
\end{tabular}

$\mathbf{E}=\mathbf{S}_{0}$. T, see Eqs. (73)-(74), does not yield any additional information. We draw on the second equation, leading to

$$
0=-\frac{\nu_{12}}{E_{11}} \sigma_{11}+\frac{1}{E_{22}} \sigma_{22}-\frac{\nu_{23}}{E_{22}} \sigma_{33} \quad \Longrightarrow \quad v_{32}=\frac{E_{22}}{\sigma_{33}}\left(-\frac{\nu_{12}}{E_{11}} \sigma_{11}+\frac{\sigma_{22}}{E_{22}}\right),
$$

where we assume that the parameters $E_{11}, E_{22}, v_{12}$ are already determined and the stresses and strains have been measured. It must be remarked that, in view of error propagation, there are sources of uncertainties. First, the measurements $\left(\sigma_{11}, \sigma_{22}, \sigma_{33}\right)$ have errors, see Fig. 6 b, c. Second, the parameters $\left(E_{11}, E_{22}, v_{12}\right)-$ from the previous identification steps - are uncertain. Thus, $\nu_{32}$ is the most sensitive quantity.

Following the procedure mentioned above, the strain measures $\varepsilon_{11}$ and $\varepsilon_{22}$ are determined using the method detailed in Appendix 3.2.1. To obtain the material parameters $E_{11}, E_{22}, v_{12}, \mu_{12}$, and $\nu_{32}$, i.e., $\kappa_{S}$ from the analytical equations (47)-(49), (53), and (54), we proceed step by step, as discussed before. In each step, we determine the material parameter just considered from the corresponding five tests (tensile tests) and four experiments (shear and compression) using a least-square method. The resulting parameters are compiled in Table 1 denoted as "analytical approach." The results of the identification using the aforementioned procedure are shown in Fig. 11.

There are a number of other possibilities to determine the parameter $v_{32}$ :

1. Instead of applying in Eq. (73) the second equation, see Eq. (54), one can draw on the third equation,

$$
\begin{aligned}
& \varepsilon_{33}=-\frac{\nu_{12}}{E_{11}} \sigma_{11}-\frac{\nu_{23}}{E_{22}} \sigma_{22}+\frac{\sigma_{33}}{E_{22}} \\
& \nu_{23}=\nu_{32}=\frac{E_{11} E_{22} \varepsilon_{33}-E_{11} \sigma_{33}+E_{22} \sigma_{11} \nu_{12}}{E_{11} \sigma_{22}} .
\end{aligned}
$$

Here, the measured axial strain $\varepsilon_{33}$ has to be considered. However, this is very sensitive since it is dependent on the machine stiffness. The sensitivity can be expressed by the derivative

$$
\frac{\partial v_{23}}{\partial \varepsilon_{33}}=\frac{E_{22}}{\sigma_{22}} .
$$

For this investigation, we have $\left|\partial v_{23} / \partial \varepsilon_{33}\right| \approx|12,964 /(-0.18)| \approx 72,000$. In other words, smallest changes in $\varepsilon_{33}$ will lead to extremely large changes in $\nu_{23}$. Thus, we cannot recommend this application. 


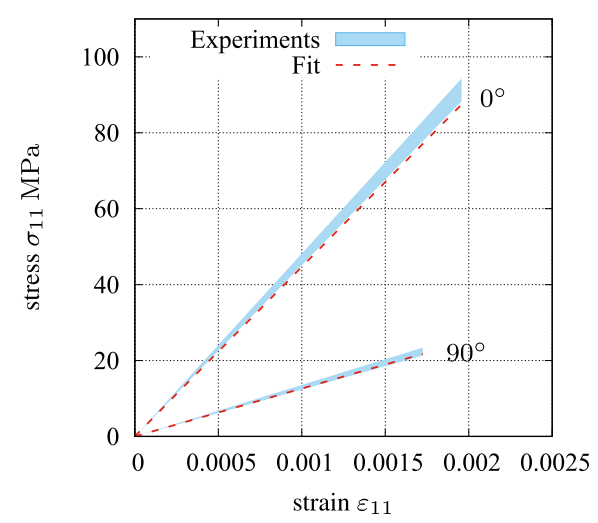

(a) Axial stress vs axial strain for $0^{\circ}$ and $90^{\circ}$ specimens

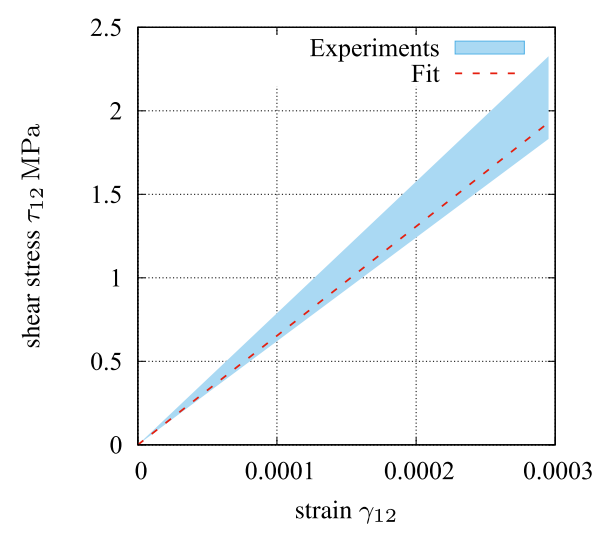

(c) Shear stress vs shear strain from shear experiment

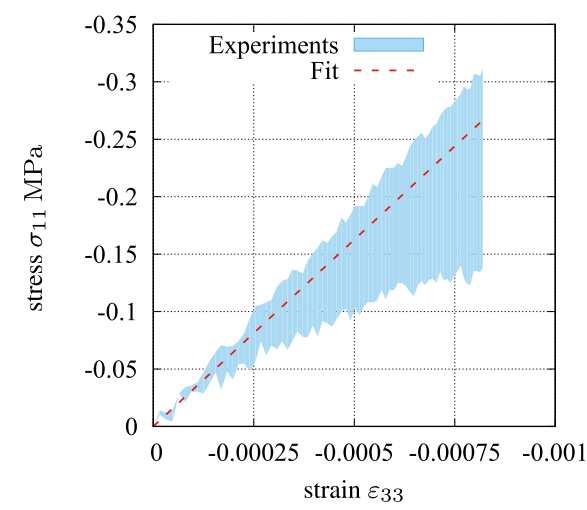

(e) Lateral stress vs axial strain of compression tests

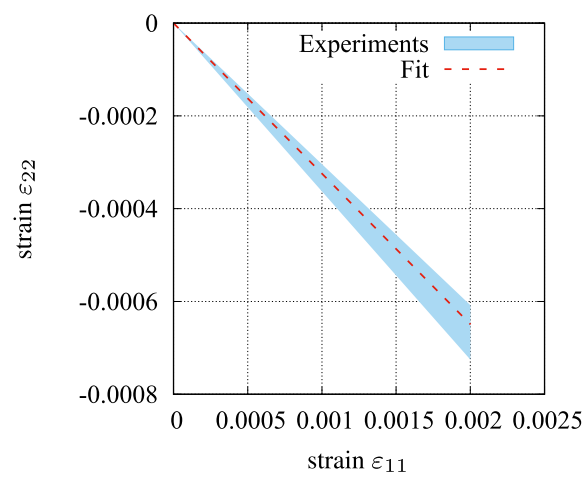

(b) Lateral strain vs axial strain for $0^{\circ}$ specimens

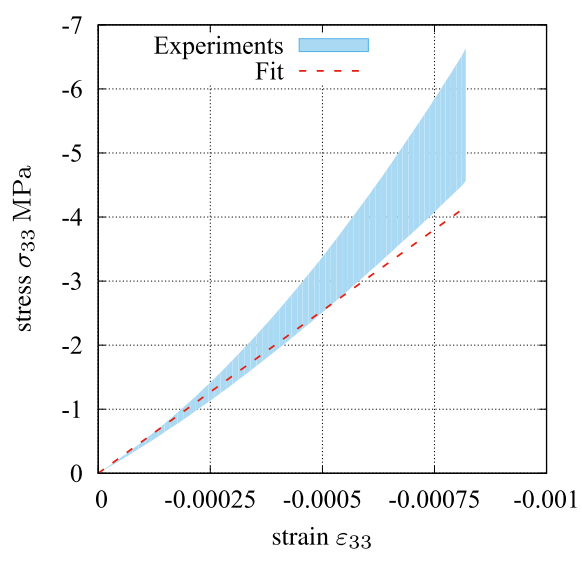

(d) Axial stress vs axial strain from compression test

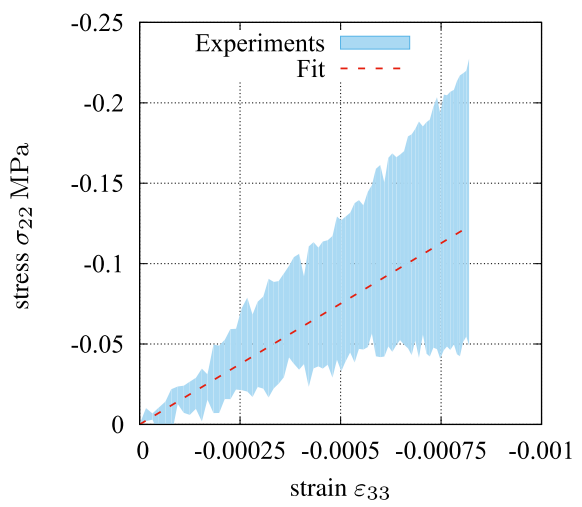

(f) Lateral stress vs axial strain of compression tests

Fig. 11 Calibrated experiment-fit diagrams using the assumption of homogeneous deformations 
2. To overcome the problem of the compression test, we reduce the number of material parameters. Let us assume the geometrical constraint $\varepsilon_{22}=\varepsilon_{33}$, which holds for a tensile test $\left(\sigma_{22}=\sigma_{33}=0\right)$ with $\gamma=0^{\circ}$ (evaluate the 2nd and 3rd equation in Eq. (73) using the compliance matrix (74)). In this case, we can derive

$$
\frac{\varepsilon_{33}}{\varepsilon_{22}}=1 \Rightarrow-\frac{\varepsilon_{33}}{\varepsilon_{22}}=-\frac{\varepsilon_{22}}{\varepsilon_{11}} \frac{\varepsilon_{11}}{\varepsilon_{22}} \frac{\varepsilon_{11}+\varepsilon_{22}}{\varepsilon_{11}+\varepsilon_{22}}=-\frac{\varepsilon_{22}}{\varepsilon_{11}}\left(\frac{1+\varepsilon_{11} / \varepsilon_{22}}{1+\varepsilon_{22} / \varepsilon_{11}}\right) .
$$

Using Eqns.(48) and (50), the relation

$$
v_{32}=v_{12}\left(\frac{1-v_{21}}{1-v_{12}}\right)
$$

is obtained. This relation is adopted in [7] without any directly discernible explanation. Together with Eq. (51), we arrive at the following estimation,

$$
v_{32}=v_{12}\left(\frac{1-v_{12} \frac{E_{22}}{E_{11}}}{1-v_{12}}\right) .
$$

The material parameters $\boldsymbol{\kappa}_{\mathrm{C}}$ and $\boldsymbol{\kappa}_{\mathrm{SP}}$ are compiled in Table 1 (Christensen formula). In this case, no nonphysical behavior is observed in a tensile computation using the set $\kappa_{\text {SP }}$. However, it is a very strong assumption. For $\kappa_{\mathrm{C}}$ and $\boldsymbol{\kappa}_{\mathrm{SP}}$, we also estimate the error propagation, see Eq. (45).

3. An alternative idea is to provide a numerical compression test using a representative volume element (RVE), see Fig. 12. The compression simulation has a fiber volume fraction of $55 \%$ and we apply $\varepsilon_{33}=1 \%$ "global" strain. Here, we take the pure resin elasticity parameters determined in Sect. 3.2.1 and the parameters of the glass fibers provided in Sect. 2.2. Using the homogenization concept proposed in [33], we determine from the nodal reaction forces at the RVE surface homogenized stresses $\bar{\sigma}_{11}, \bar{\sigma}_{22}$, and $\bar{\sigma}_{33}$, which are required in Eq. (54). This leads to $\nu_{32}$ in Table 1 (synthetic compression test), and the estimation of $\kappa_{\mathrm{C}}$ and $\kappa_{\mathrm{SP}}$. These parameters are now on a similar order of magnitude. Moreover, for the latter, the error propagation scheme Eq. (45) is chosen again.

4. Let us consider a combination of the mixture rules, see [32], with particular attention to the HalpinTsai estimations, see [11]. With a fiber volume fraction $\Phi_{f}=0.55$, the Young's moduli for the fiber $E_{f}=73000 \mathrm{MPa}$, and the resin (matrix material) $E_{m}=2510 \mathrm{MPa}$, we obtain

$$
E_{11}=E_{f} \Phi_{f}+E_{m}\left(1-\Phi_{f}\right) \approx 41280 \mathrm{MPa} .
$$

Similarly, the Poisson's number $v_{12}$ with $v_{f}=0.22$ and $v_{m}=0.39$ can be determined

$$
v_{12}=v_{f} \Phi_{f}+v_{m}\left(1-\Phi_{f}\right) \approx 0.3 .
$$

The transverse Young's modulus $E_{22}$ can be estimated by the Halpin-Tsai formula, see [11,32]:

$$
E_{22}=E_{m} \frac{1+\varphi_{1} \eta_{1} \Phi_{f}}{1-\eta_{1} \Phi_{f}}
$$

with

$$
\varphi_{1}=2+40 \Phi_{f}^{10}, \quad \text { and } \eta_{1}=\frac{E_{f} / E_{m}-1}{E_{f} / E_{m}+\varphi_{1}}
$$

for circular fiber cross sections. Here, we obtain with $\varphi_{1} \approx 2.1$ and $\eta_{1} \approx 0.9$ the value $E_{22} \approx 10149 \mathrm{MPa}$. Using Christensen's formula (59) again, one obtains $\nu_{32}=0.39$. Finally, the shear modulus $\mu_{12}$ can be estimated using another Halpin-Tsai formula,

$$
\mu_{12}=\mu_{m} \frac{1+\varphi_{2} \eta_{2} \Phi_{f}}{1-\eta_{2} \Phi_{f}}
$$

with

$$
\varphi_{2}=1+40 \Phi_{f}^{10}\left(\text { for circular fibers), and } \eta_{2}=\frac{\mu_{f} / \mu_{m}-1}{\mu_{f} / \mu_{m}+\varphi_{2}}\right.
$$




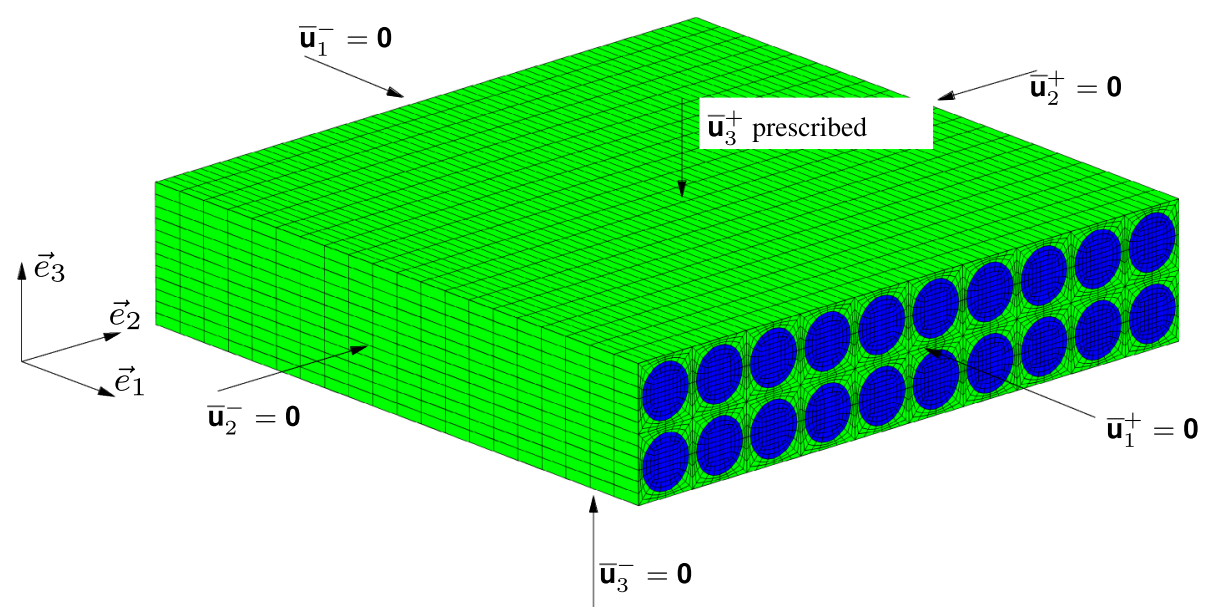

Fig. 12 Finite element meshes (20-noded hexahedral elements) for a glass fiber volume fraction of 55\%

$$
\mu_{f}=\frac{E_{f}}{2\left(1+v_{f}\right)}, \quad \mu_{m}=\frac{E_{m}}{2\left(1+v_{m}\right)} .
$$

This leads to $\varphi_{2}=1.1, \eta_{2}=0.94$, and $\mu_{12}=2928 \mathrm{MPa}$. The values $E_{11}, E_{22}, v_{12}, \nu_{23}$, and $\mu_{12}$ are in the order of values of the second and third column of Table 1 . However, the stiffnesses $E_{11}, E_{22}$, and $\mu_{12}$ are below the values obtained from the other procedures, which are based on the experimental results of the composite material.

\subsubsection{Synthetic data}

The goal of this section is to show that local identifiability is not given if only particular DIC information is considered. In order not to mix up disturbed, real measurement data with the concept of identifiability of material parameters, we use the concept of re-identification. Here, finite element calculations are performed with given material parameters and the generated nodal displacements are considered as measurement data. This information is then used to solve the identification problem, which should lead to the expected material parameters, i.e., those which are used to generate the data. The determinant of the Hessian matrix (38) then provides information about the identifiability of the parameters.

\section{Re-identification using $0^{\circ}, 45^{\circ}$, and $90^{\circ}$ DIC data}

As a first attempt to check for unique identifiability of the parameters, using the parameters $\boldsymbol{\kappa}_{\mathrm{SP}}$, synthetic data (force and DIC data) are created. Using these data and random starting guess values of the parameters for the nonlinear optimizer, we try to re-identify the analytically calculated parameters. However, it was observed that the material parameters obtained cannot be re-identified. In this case, $\operatorname{det} \boldsymbol{H}=7.4 e-26 \approx 0$ holds, which confirms that the parameters are not uniquely identifiable. In other words, if only in-plane information is chosen, no reliable parameters can be determined. This is already supported by the preliminary analytical investigations.

\section{Re-identification using $0^{\circ}, 45^{\circ}, 90^{\circ}$, and shear DIC data}

As a second possibility, in addition to the $0^{\circ}$ and $90^{\circ}$ DIC data as well as $45^{\circ}$ data, synthetic data for shear tests were also created in order to check the identifiability of the parameters. It was found out that det $\boldsymbol{H}=$ $8.5 e-24 \approx 0$ in this case. This means that, in order to identify the parameters, one more test, e.g., the compression test, is required to uniquely identify the parameters. 


\section{Re-identification using $0^{\circ}, 90^{\circ}$, shear DIC data, and compression test}

As a different possibility, synthetic data of compression tests as explained in Sect. 2.2.3 were also included in addition to force and DIC data of $0^{\circ}, 90^{\circ}$, and shear tests in order to re-identify the material parameters. In this case, $\operatorname{det} \boldsymbol{H}=0.69 \neq 0$-i.e., it is possible to re-identify the material parameters. This confirms that in order to uniquely identify the material parameters, it is essential to first investigate experimental tests that are required for identifiability. Different tests address different parameters within a material model. Sometimes, performing different tests does not mean that different parameters are addressed. For example, the $45^{\circ}$ and the shear tests address the same parameters.

\subsubsection{DIC/FEM approach (transverse isotropy)}

A different approach to identify the material parameters is given by assuming inhomogeneous deformations. In this case, we have to draw on the original DIC information. On the basis of the strain determination concept proposed in [16], the principal strains are determined on the basis of both the DIC data and the finite element nodal information, see the discussion in the previous sections as well. Additionally, the force data of the testing machine are taken into account. In the following, three approaches are investigated using the leastsquare method based on full-field surface information and finite element simulations. Since the compression tests are not reliable, the parameter $v_{32}$ can be determined either by Christensen's approach, see Eq. (59), or by the numerical homogenization technique of the compression test, see Fig. 12. The latter approach consistently leads to a pure homogenization technique of the four basis tests (tension $\gamma=0^{\circ}, 90^{\circ}$, shear, and compression) using the RVE in Fig. 12.

1. DIC/FEM and prescription of $\nu_{32}$ : Here, we draw on the force and DIC data of the tensile tests for $0^{\circ}$ and $90^{\circ}$, the shear tests, and fixing the $v_{32}=0.39$ (obtained from analytical calculations). Since the finite element implementation is based on the usage of the parameter set $\kappa_{\mathrm{SP}}$, we have to formulate an equality constraint condition, see Eq. (87), with

$$
c_{I}\left(\kappa_{\mathrm{SP}}\right)=v_{32}-\left(\frac{\alpha^{2}-\Lambda\left(\beta+4 \mu_{L}-2 \mu_{T}\right)}{(\alpha+\Lambda)^{2}-\left(\Lambda+2 \mu_{T}\right)\left(2 \alpha+\beta+\Lambda+4 \mu_{L}-2 \mu_{T}\right)}\right)=0,
$$

see Eq. (18) $)_{2}$. The resulting parameters are assembled in Table 2 (FEM/DIC ( $\nu_{32}$ fixed)). Obviously, the confidence interval of $\alpha$ is larger than the value itself. Similarly, this applies to the parameter $\Lambda$, where the confidence interval is of the same order as the value itself. However, if we look at the magnitude of the parameters and their confidence intervals, they are comparable to Table 1 (analytical approach), or even better, to the parameter set $\boldsymbol{\kappa}_{\mathrm{C}}$ for both procedures. Remember that in the analytical investigations, $\boldsymbol{\kappa}_{\mathrm{S}}$ were identified and $\kappa_{\mathrm{SP}}$ was estimated with error propagation. Here, it has been carried out in reverse.

As secondary information, the following should be noted: In the case of IND, the computational time required is around $224 \mathrm{~s}$, while END takes around $752 \mathrm{~s}$. In other words, we can save $70 \%$ of the computational times. This depends on the choice of the initial guess of the parameters. Here, we have chosen: $\alpha=300 \mathrm{~N} \mathrm{~mm}^{-2}, \beta=30,000 \mathrm{~N} \mathrm{~mm}^{-2}, \mu_{T}=5000 \mathrm{~N} \mathrm{~mm}^{-2}, \Lambda=5000 \mathrm{~N} \mathrm{~mm}^{-2}$, and $\mu_{L}=5000 \mathrm{~N} \mathrm{~mm}^{-2}$.

2. Since a fixed $\nu_{32}$ is a very strong assumption, we again consider a numerical homogenization scheme for the compression test, see Fig. 12. In other words, we have five DIC data sets for each tension test $\left(\gamma=0^{\circ}, 90^{\circ}\right)$, and four for shear, and assume one data set for RVE using the elasticity properties for the pure resin and the glass fibers. In this case, we arrive at the parameters of Table 2 (FEM/DIC (RVE)). These are now very similar to the results of prescribed $\nu_{32}$, see the discussion before.

3. Since we applied the compression test from an RVE, why should we not use the RVE to generate results of the tension test $\left(\gamma=0^{\circ}, 90^{\circ}\right)$, shear and compression. Using the least-square/FEM approach, we obtain the parameters in the "FEM (RVE)" column of Table 2. There, only the shear modulus $\mu_{L}$ deviates more from all previous investigations. Thus, much better RVEs-resulting from $\mu$-CT data (not available for these investigations), for example—are required to investigate this.

\subsubsection{Summary of parameter identification approaches}

We discussed several approaches to identify the 5 material parameters of transverse isotropy for the case of small strains and linear elasticity in detail. This is summarized in the following. The main goal here is the 
Table 2 Determination of $\kappa_{\mathrm{SP}}$ and calculation of $\kappa_{\mathrm{C}}$ and $\kappa_{\mathrm{S}}$ using the assumption of inhomogeneous deformations

\begin{tabular}{|c|c|c|c|c|}
\hline Parameter & Dimension & FEM/DIC ( $v_{32}$ fixed $)$ & FEM/DIC (RVE) & FEM (RVE) \\
\hline \multicolumn{5}{|l|}{$\kappa_{\mathrm{SP}}$} \\
\hline$\alpha$ & $\mathrm{N} \mathrm{mm}^{-2}$ & $190 \pm 225$ & $200 \pm 261$ & 173 \\
\hline$\beta$ & $\mathrm{N} \mathrm{mm}^{-2}$ & $28,014 \pm 4884$ & $28,200 \pm 4820$ & 35,402 \\
\hline$\mu_{T}$ & $\mathrm{~N} \mathrm{~mm}^{-2}$ & $3865 \pm 143$ & $3871 \pm 127$ & 4196 \\
\hline$\Lambda$ & $\mathrm{N} \mathrm{mm}^{-2}$ & $5772 \pm 1628$ & $5146 \pm 1833$ & 5537 \\
\hline$\mu_{L}$ & $\mathrm{~N} \mathrm{~mm}^{-2}$ & $3514 \pm 3$ & $3518 \pm 22$ & 2933 \\
\hline \multicolumn{5}{|l|}{$\kappa_{\mathrm{C}}$} \\
\hline$C_{11}$ & $\mathrm{~N} \mathrm{~mm}^{-2}$ & $40,492 \pm 5175$ & $40,077 \pm 5190$ & 44,452 \\
\hline$C_{22}$ & $\mathrm{~N} \mathrm{~mm}^{-2}$ & $13,502 \pm 1652$ & $12,889 \pm 1851$ & 13,756 \\
\hline$C_{12}$ & $\mathrm{~N} \mathrm{~mm}^{-2}$ & $5962 \pm 1643$ & $5345 \pm 1852$ & 5537 \\
\hline$C_{23}$ & $\mathrm{~N} \mathrm{~mm}^{-2}$ & $5772 \pm 1628$ & $5146 \pm 1833$ & 5365 \\
\hline$C_{66}$ & $\mathrm{~N} \mathrm{~mm}^{-2}$ & $3514 \pm 3$ & $3518 \pm 22$ & 2933 \\
\hline \multicolumn{5}{|l|}{$\kappa_{\mathrm{S}}$} \\
\hline$E_{11}$ & $\mathrm{~N} \mathrm{~mm}^{-2}$ & $36,803 \pm 4898$ & $36,908 \pm 4839$ & 41,245 \\
\hline$E_{22}$ & $\mathrm{~N} \mathrm{~mm}^{-2}$ & $10,726 \pm 553$ & $10,562 \pm 627$ & 11,394 \\
\hline$v_{12}$ & - & $0.31 \pm 0.03$ & $0.29 \pm 0.04$ & 0.28 \\
\hline$\mu_{12}$ & $\mathrm{~N} \mathrm{~mm}^{-2}$ & $3514 \pm 3$ & $3518 \pm 22$ & 2933 \\
\hline$v_{32}$ & - & $0.39 \pm 0.05$ & $0.36 \pm 0.07$ & 0.36 \\
\hline
\end{tabular}

unique identification of the parameters and the necessary experiments. For this discussion, we separate $\boldsymbol{\kappa}_{\mathrm{S}}$ into $\hat{\boldsymbol{\kappa}}_{\mathrm{s}}=\left\{E_{11}, E_{22}, \nu_{12}, \mu_{12}\right\}$ and $\nu_{32}$.

1. (Pure analytical identification of $\boldsymbol{\kappa}_{\mathrm{S}}$ ) From the experimental side lateral and axial strains from tensile tests $\left(\gamma=0^{\circ}, 90^{\circ}\right)$, a shear experiment, and a special compression test is required. The latter has to be treated very carefully since it is very hard to obtain reliable results.

2. (Pure analytical identification with Christensen's formulae (59) to determine $v_{32}$ ) To circumvent the special compression test, a rough estimation of the material parameter $v_{32}$ can be performed. All other parameters $\hat{\boldsymbol{\kappa}}_{\mathrm{S}}$ are determined by the two tensile tests, and the shear test. This leads in our applications to appropriate results.

3. (Analytical identification of $\hat{\boldsymbol{\kappa}}_{\mathrm{S}}$ and numerical determination of $\nu_{32}$ using an RVE) To circumvent the compression test with a special die/punch device, a representative volume element can be chosen, which is loaded with the boundary conditions of the compression test (laterally bounded). The smeared lateral stresses can be evaluated to determine $\nu_{32}$. The remaining parameters $\hat{\boldsymbol{\kappa}}_{\mathrm{s}}$ are determined by the tensile and shear tests as discussed before.

Approaches 2 and 3 lead to similar results. Approach 1 depends essentially on the reliability of the sensitive compression test.

To circumvent all the four different tests mentioned afore, one pure tensile test of the matrix material to determine Young's modulus and the Poisson number is an alternative. In this case, particular homogenization methods must be treated.

4. (Pure analytical homogenization) Using mixture rules of [11] in combination of Christensen's formulae (59) requires only the elastic material parameters of the two constituents (matrix material and fiber material). But these parameters deviate considerably from the parameters found before.

5. (Fully numerical consideration of RVE) A representative volume element is subjected to uniaxial tension for $\gamma=0^{\circ}, 90^{\circ}$, shear, and compression. This leads very similar results to pure analytical homogenization results.

\section{Conclusions}

In this article, several approaches to determine the material parameters of a model of linear elastic, small strain, transverse isotropy for unidirectional fiber composite materials are proposed. The experiments for uniaxial tension and shear are provided using a tensile testing machine. The use of DIC data provides the full-field deformation information, showing effects such as rigid body motions in the testing machine, and insufficient straight alignment of the testing machine (bending of the specimens). We can observe imperfections in the 
clamping, local inhomogeneities in the specimens due to the fiber bundles, small air inclusions, and so forth. From these test data, we have to identify the material parameters. In more modern approaches, the DIC data are chosen to identify material parameters, and an approach of using DIC in combination with finite elements will lead to some parameters. However, it turns out that pure in-plane information cannot provide reliable material parameters. The approach does not overcome the general question whether the material parameter can be addressed. Thus, the concept of local identifiability is applied on the basis of generating synthetic data and re-identification evaluating the Hessian matrix. This is a quite suitable indicator of whether the parameters are uniquely identifiable. It is shown that a compression test is required. However, the newly built compression device shows a response which cannot be explained in terms of the results that could be expected from theoretical conclusions. Thus, various identification approaches are followed. First, purely analytical results under the assumption of homogeneous deformations indicate that out-of-plane information is required. Thus, the compression test was chosen. This leads to the first conclusion that the DIC/FEM approach to identify material parameters is not a general tool to determine parameters. A detailed knowledge of the identifiability of the material parameters should be known in advance. Apart from the purely analytical identification, a mixture is chosen based on an RVE instead of the compression test data. However, this requires the material parameters of the fiber material and the pure resin. This is done in a similar manner using DIC data combined with the RVE approach for the compression test. This leads to similar material parameters as for the analytical approach. A second conclusion is related to providing the confidence interval of the material parameters indicating the quality of the parameters. A third conclusion concerns constitutive models, which can be expressed by different sets of material parameters. Although one can identify one set of parameters, the dependent set of parameters can be very uncertain due to simple error propagation investigations. This can lead to completely unphysical results in computations. A third concept is based on purely homogenized RVEs, which is a common approach in micro-to-macro-transitions of heterogeneous materials. It leads to parameters that are in a similar order to the other set of parameters, although only a rough RVE is chosen.

Apart from the conceptual approach, we compare two numerical concepts, namely internal and external numerical differentiation. It is very common to determine the sensitivity matrices in gradient-based optimization schemes by numerical differentiation, which is very costly. Alternatively, we provide the tangent computations in a purely analytical approach. This can save up to $70 \%$ of computational time.

In conclusion, it should be noted that material parameter identification is a discipline by itself, and extensive knowledge about the constitutive model, the theory of finite elements, experimental uncertainties, and so forth is required. A simple plug-and-play procedure is not available, as shown for a pure elastic constitutive model for small strains.

Acknowledgements We would like to thank Mrs. W. Pangboonyanon of the Institute of Polymer Materials and Plastics Engineering at Clausthal University of Technology for producing the composite plates. Furthermore, we gratefully acknowledge the financial support provided by the government of Lower Saxony, Germany, for the P2-project in the doctoral program "Selforganizing multifunctional structures for adaptive high performance light-weight constructions," which is located at the Campus Funktionswerkstoffe of the Clausthal University of Technology, and the German Research Foundation project, DFG HA 2024/19-1.

Funding Open Access funding enabled and organized by Projekt DEAL.

Open Access This article is licensed under a Creative Commons Attribution 4.0 International License, which permits use, sharing, adaptation, distribution and reproduction in any medium or format, as long as you give appropriate credit to the original author(s) and the source, provide a link to the Creative Commons licence, and indicate if changes were made. The images or other third party material in this article are included in the article's Creative Commons licence, unless indicated otherwise in a credit line to the material. If material is not included in the article's Creative Commons licence and your intended use is not permitted by statutory regulation or exceeds the permitted use, you will need to obtain permission directly from the copyright holder. To view a copy of this licence, visit http://creativecommons.org/licenses/by/4.0/.

\section{A Alternative representation of Voigt matrices}

The Voigt representation of the fourth-order tensor (Spencer's model) is chosen for the component representation. This $(6 \times 6)$ matrix representation reads as follows. The stress-strain relation is given by

$$
\mathbf{T}=\mathbf{C}_{\gamma} \mathbf{E}
$$

with $\mathbf{T} \in \mathbb{R}^{6}, \mathbf{E} \in \mathbb{R}^{6}$, and $\mathbf{C}_{\gamma} \in \mathbb{R}^{6 \times 6}$. ( $\gamma$ indicates the fiber orientation angle for plane problems.) In a finite element implementation, an elasticity matrix $\mathbf{C}_{\gamma}$ with arbitrary fiber directions a has to be implemented, see, 
for example, [15]. If we stay on the level of purely analytical considerations, which is the case for homogeneous deformations, it is common to take the following representation $\left(\mathbf{a}=\mathbf{e}_{1}\right)$

$$
\mathbf{a}=\mathbf{e}_{1}: \underbrace{\left\{\begin{array}{l}
\sigma_{11} \\
\sigma_{22} \\
\sigma_{33} \\
\tau_{12} \\
\tau_{23} \\
\tau_{31}
\end{array}\right\}}_{\mathbf{T}}=\underbrace{\left[\begin{array}{cccccc}
\Lambda-2 \mu_{T}+2 \alpha+4 \mu_{L}+\beta & \Lambda+\alpha & \Lambda+\alpha & 0 & 0 & 0 \\
\Lambda+\alpha & \Lambda+2 \mu_{T} & \Lambda & 0 & 0 & 0 \\
\Lambda+\alpha & \Lambda & \Lambda+2 \mu_{T} & 0 & 0 & 0 \\
0 & 0 & 0 & \mu_{L} & 0 & 0 \\
0 & 0 & 0 & 0 & \mu_{T} & 0 \\
0 & 0 & 0 & 0 & 0 & \mu_{L}
\end{array}\right]}_{\mathbf{C}_{0^{\circ}}} \underbrace{\left\{\begin{array}{l}
\varepsilon_{11} \\
\varepsilon_{22} \\
\varepsilon_{33} \\
\gamma_{12} \\
\gamma_{23} \\
\gamma_{31}
\end{array}\right\},}_{\mathbf{E}}
$$

$\left(\gamma_{i j}=2 E_{i j}\right)$. Christensen [8] only takes this representation to discuss various loading directions. In other words, the loading direction is changed, but not the orientation vector a, i.e., the elasticity matrix in Eq. (69) is constant. The index $\gamma$ of the elasticity matrix $\mathbf{C}_{\gamma}$ indicates the angle between the loading direction $\mathbf{e}_{1}$ in the uniaxial tensile test and the fiber direction $\mathbf{a}$ of the specimen, $\mathbf{a} \cdot \mathbf{e}_{1}=\cos \gamma, \gamma=\arccos \left(\mathbf{a} \cdot \mathbf{e}_{1}\right)$, see Fig. $1 \mathrm{~b}$. Alternative expressions of the components of the elasticity matrix $\mathbf{C}-$ not applying "Spencer's material parameters" $\kappa_{\mathrm{SP}}=\left\{\Lambda, \mu_{L}, \alpha, \beta, \mu_{T}\right\}$-are shown in the following. These are the parameter sets $\kappa_{\mathrm{C}}=\left\{C_{11}, C_{22}, C_{12}, C_{23}, C_{66}\right\}$, and $\kappa_{\mathrm{S}}=\left\{E_{11}, E_{22}, \nu_{21}, \nu_{32}, \mu_{23}\right\}$. The representation of $\kappa_{\mathrm{C}}$ is provided for completeness of the presentation.

The elasticity matrices $\mathbf{C} \in \mathbb{R}^{6 \times 6}$ for specific fiber orientations and material parameter sets can also be written, for example, in the following representation using the parameter set $\kappa_{\mathrm{C}}$,

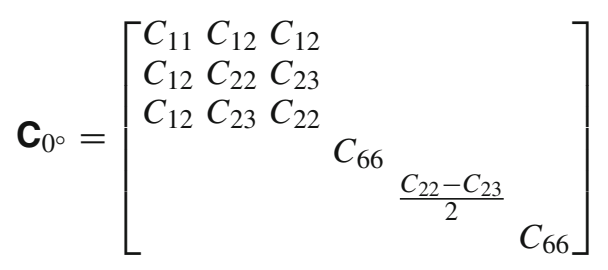

for a fiber orientation angle $\gamma=0^{\circ}$. Comparing representation (70) with (69), i.e., the parameter set $\kappa_{\text {SP }}$, yields the identities

$$
C_{11}=\Lambda+2 \mu_{T}+2 \alpha+4\left(\mu_{L}-\mu_{T}\right)+\beta, \quad C_{22}=\Lambda+2 \mu_{T}, \quad C_{12}=\Lambda+\alpha, \quad C_{23}=\Lambda, \quad C_{66}=\mu_{L}
$$

or, vice versa,

$$
\alpha=C_{12}-C_{23}, \quad \beta=C_{11}-2 C_{12}+C_{22}-4 C_{66}, \quad \Lambda=C_{23}, \quad \mu_{L}=C_{66}, \quad \mu_{T}=\frac{C_{22}-C_{23}}{2} .
$$

A third possibility for the matrix representation (68), i.e., the concrete expressions (69) or (70), is obtained by the compliance matrix $\mathbf{S} \in \mathbb{R}^{6 \times 6}, \mathbf{E}=\mathbf{S T}, \mathbf{C}=\mathbf{S}^{-1}$, see [8]. For $\gamma=0^{\circ}$, we have

$$
\mathbf{E}=\mathbf{S}_{0^{\circ}} \mathbf{T}
$$

with

$$
\mathbf{S}_{0^{\circ}}=\left[\begin{array}{cccccc}
\frac{1}{E_{11}} & -\frac{v_{21}}{E_{22}} & -\frac{\nu_{21}}{E_{22}} & & & \\
-\frac{v_{12}}{E_{11}} & \frac{1}{E_{22}} & -\frac{v_{32}}{E_{22}} & & & \\
-\frac{\nu_{12}}{E_{11}} & -\frac{\nu_{23}}{E_{22}} & \frac{1}{E_{22}} & & & \\
& & & \frac{1}{\mu_{12}} & & \\
& & & & \frac{1}{\mu_{23}} & \\
& & & & \frac{1}{\mu_{12}}
\end{array}\right]=\left[\begin{array}{lll}
\mathbf{S}_{0^{\circ}}^{u} & \\
& \mathbf{S}_{0^{\circ}}^{l}
\end{array}\right]
$$

$\mathbf{S}_{0^{\circ}}^{u} \in \mathbb{R}^{3 \times 3}, \mathbf{S}_{0^{\circ}}^{l} \in \mathbb{R}^{3 \times 3}$. This leads to

$$
\mathbf{C}_{0^{\circ}}=\left[\begin{array}{lll}
\mathbf{S}_{0^{\circ}}^{u}-1 & & \\
& \mathbf{S}_{0^{\circ}}^{l}-1
\end{array}\right]
$$


with

$$
\mathbf{S}_{0^{\circ}}^{u-1}=\frac{1}{\delta}\left[\begin{array}{ccc}
E_{11}\left(v_{23} v_{32}-1\right) & E_{11} v_{21}\left(1+v_{23}\right) & E_{11} v_{21}\left(1+v_{32}\right) \\
E_{22} v_{12}\left(1+v_{32}\right) & E_{22}\left(v_{12} v_{21}-1\right) & E_{22}\left(v_{12} v_{21}+v_{32}\right) \\
E_{22} v_{12}\left(1+v_{23}\right) & E_{22}\left(v_{12} v_{21}+v_{23}\right) & E_{22}\left(v_{12} v_{21}-1\right)
\end{array}\right],
$$

$\delta=-1+v_{23} v_{32}+v_{12} v_{21}\left(2+v_{23}-v_{32}\right)$, and

$$
\mathbf{S}_{0^{\circ}}^{l-1}=\left[\begin{array}{lll}
\mu_{12} & & \\
& \mu_{23} & \\
& & \mu_{12}
\end{array}\right] .
$$

In this case, we have the third parameter set $\kappa_{S}$. To complete the dependencies, we calculate:

$$
\begin{aligned}
& E_{11}=C_{11}-\frac{2 C_{12}^{2}}{C_{22}+C_{23}}, \quad E_{22}=\frac{\left(C_{22}-C_{23}\right)\left(C_{11}\left(C_{22}+C_{23}\right)-2 C_{12}^{2}\right)}{C_{11} C_{22}-C_{12}^{2}}, \\
& \nu_{21}=\frac{C_{12}\left(C_{23}-C_{22}\right)}{C_{12}^{2}-C_{11} C_{22}}, \quad v_{32}=\frac{C_{12}^{2}-C_{11} C_{23}}{C_{12}^{2}-C_{11} C_{22}}, \quad \mu_{23}=\frac{C_{22}-C_{23}}{2}, \\
& C_{11}=\frac{E_{11} E_{22}\left(v_{32}-1\right)}{2 E_{11} v_{21}^{2}+E_{22}\left(v_{32}-1\right)}, \quad C_{22}=\frac{E_{22}\left(E_{11} v_{21}^{2}-E_{22}\right)}{\left(v_{32}+1\right)\left(2 E_{11} v_{21}^{2}+E_{22}\left(v_{32}-1\right)\right)}, \quad C_{66}=\mu_{12}, \\
& C_{12}=\frac{E_{11} E_{22} v_{21}}{-2 E_{11} v_{21}^{2}-E_{22} v_{32}+E_{22}}, \quad C_{23}=-\frac{E_{22}\left(E_{11} v_{21}^{2}+E_{22} v_{32}\right)}{\left(v_{32}+1\right)\left(2 E_{11} v_{21}^{2}+E_{22}\left(v_{32}-1\right)\right)}, \\
& \Lambda=-\frac{E_{22}\left(E_{11} v_{21}^{2}+E_{22} v_{32}\right)}{\left(1+v_{32}\right)\left(2 E_{11} v_{21}^{2}+E_{22}\left(v_{32}-1\right)\right)}, \quad \alpha=\frac{E_{22}\left(E_{11} v_{21}\left(v_{21}-v_{32}-1\right)+E_{22} v_{32}\right)}{\left(1+v_{32}\right)\left(2 E_{11} v_{21}^{2}+E_{22}\left(v_{32}-1\right)\right)}, \\
& \beta=E_{11}-\frac{\left(E_{22}-2 E_{11} \nu_{21}\right)^{2}}{4 E_{11} v_{21}^{2}+2 E_{22}\left(\nu_{32}-1\right)}+\frac{E_{22}}{2\left(1+v_{32}\right)}-4 \mu_{23}, \quad \mu_{T}=\frac{E_{22}}{2\left(1+v_{32}\right)}, \quad \mu_{L}=\mu_{12} \text {, } \\
& E_{11}=-\frac{(\alpha+\Lambda)^{2}}{\Lambda+\mu_{T}}+2 \alpha+\beta+\Lambda+4 \mu_{L}-2 \mu_{T} \\
& E_{22}=\frac{2 \mu_{T}\left(2\left(\Lambda+\mu_{T}\right)\left(2 \alpha+\beta+\Lambda+4 \mu_{L}-2 \mu_{T}\right)-2(\alpha+\Lambda)^{2}\right)}{\left(\Lambda+2 \mu_{T}\right)\left(2 \alpha+\beta+\Lambda+4 \mu_{L}-2 \mu_{T}\right)-(\alpha+\Lambda)^{2}}, \\
& v_{21}=-\frac{2 \mu_{T}(\alpha+\Lambda)}{(\alpha+\Lambda)^{2}-\left(\Lambda+2 \mu_{T}\right)\left(2 \alpha+\beta+\Lambda+4 \mu_{L}-2 \mu_{T}\right)}, \\
& v_{32}=\frac{\alpha^{2}-\Lambda\left(\beta+4 \mu_{L}-2 \mu_{T}\right)}{(\alpha+\Lambda)^{2}-\left(\Lambda+2 \mu_{T}\right)\left(2 \alpha+\beta+\Lambda+4 \mu_{L}-2 \mu_{T}\right)}, \\
& \mu_{23}=\mu_{T} \text {. }
\end{aligned}
$$

\section{B Symmetry properties of elasticity relation}

The elasticity relation (1) and the fourth-order elasticity tensor (2) are consistently derived by applying the Gateaux derivative on the specific strain energy function (hyperelasticity)

$$
\psi(\mathbf{E}, \mathbf{M})=\hat{\psi}\left(\mathrm{I}_{\mathbf{E}}, \mathrm{II}_{\mathbf{E}}, \mathrm{IV}_{\mathbf{E}}, \mathrm{V}_{\mathbf{E}}\right)=\frac{\Lambda}{2} \mathrm{I}_{\mathbf{E}}^{2}+\mu_{T} \mathrm{II}_{\mathbf{E}}+\alpha \mathrm{I}_{\mathbf{E}} \mathrm{IV}_{\mathbf{E}}+\frac{\beta}{2} \mathrm{IV}_{\mathbf{E}}^{2}+2\left(\mu_{L}-\mu_{T}\right) \mathrm{V}_{\mathbf{E}},
$$

with

$$
\mathrm{D}_{\mathbf{E}} \psi(\mathbf{E}, \mathbf{M})[\mathbf{H}]=\frac{\partial \psi(\mathbf{E}, \mathbf{M})}{\partial \mathbf{E}} \cdot \mathbf{H} \quad \text { with } \quad \mathbf{D}_{\mathbf{E}} \psi(\mathbf{E}, \mathbf{M})[\mathbf{H}]=\left.\frac{\mathrm{d}}{\mathrm{d} \lambda} \psi(\mathbf{E}+\lambda \mathbf{H}, \mathbf{M})\right|_{\lambda=0}
$$


i.e., $\mathbf{T}=\mathbf{h}(\mathbf{E}, \mathbf{M})=\frac{\partial \psi(\mathbf{E}, \mathbf{M})}{\partial \mathbf{E}}$ holds, and the property $\mathbf{E}^{T}=\mathbf{E}$ was evaluated. (The explicit dependence on the density is omitted for brevity and is considered to be included in the material parameters, see for the theory of hyperelasticity, for example, [22].)

The fourth-order elasticity tensor $\mathcal{C}$ in Eq. (2) can be derived either from Eq. (1) using the transposition rule (change of the second and third index) $[\mathbf{A} \otimes \mathbf{B}]^{T_{23}} \mathbf{C}=\mathbf{A C B}^{T}$, or by evaluating the Gateaux derivative

$$
\mathcal{C} \mathbf{H}=\frac{\partial \mathbf{h}(\mathbf{E}, \mathbf{M})}{\partial \mathbf{E}} \mathbf{H}=\mathrm{D}_{\mathbf{E}} \mathbf{h}(\mathbf{E}, \mathbf{M})[\mathbf{H}]=\left.\frac{\mathrm{d}}{\mathrm{d} \lambda} \mathbf{h}(\mathbf{E}+\lambda \mathbf{H}, \mathbf{M})\right|_{\lambda=0} .
$$

This is symbolically equivalent to

$$
\mathcal{C}=\frac{\partial \mathbf{h}(\mathbf{E}, \mathbf{M})}{\partial \mathbf{E}}=\frac{\partial^{2} \psi(\mathbf{E}, \mathbf{M})}{\partial \mathbf{E} \partial \mathbf{E}}, \quad c_{i j k l}=\frac{\partial h_{i j}}{\partial E_{k l}}=\frac{\partial^{2} \psi}{\partial E_{i j} \partial E_{k l}},
$$

and implies the major symmetries $\mathcal{C}=\mathcal{C}^{T}$ with $\mathcal{C}^{T}=c_{k l i j} \mathbf{e}_{i} \otimes \mathbf{e}_{j} \otimes \mathbf{e}_{k} \otimes \mathbf{e}_{l}$. The major symmetry is guaranteed, which is especially valid for the terms $\mathcal{C}^{(2)}=\mathcal{I}=[\mathbf{I} \otimes \mathbf{I}]^{T_{23}}$ and $\mathcal{C}^{(5)}=[\mathbf{I} \otimes \mathbf{M}+\mathbf{M} \otimes \mathbf{I}]^{T_{23}}$. However, the commonly assumed minor symmetry, $c_{i j k l}=c_{i j l k}$ is not embedded in this derivation of applying the Gateaux derivative and the evaluation of $\mathbf{E}^{T}=\mathbf{E}$.

To circumvent this minor lack, we assume that the specific strain energy function to be dependent on the symmetric part of the strain tensor $\mathbf{E}, \mathbf{E}_{\text {sym }}=\left(\mathbf{E}+\mathbf{E}^{T}\right) / 2=\overline{\mathcal{I}} \mathbf{E}$ using the symmetrizer $\overline{\mathcal{I}}:=1 / 2(\mathcal{I}+\hat{\mathcal{I}})$ with $\hat{\mathcal{I}}=[\mathbf{I} \otimes \mathbf{I}]^{T_{24}}$. Here, we have the transposition of the second and fourth index $\hat{\mathcal{I}}=\delta_{i l} \delta_{k j} \mathbf{e}_{i} \otimes \mathbf{e}_{j} \otimes \mathbf{e}_{k} \otimes \mathbf{e}_{l}$ with the general property $[\mathbf{A} \otimes \mathbf{B}]^{T_{24}} \mathbf{C}=\mathbf{A} \mathbf{C}^{T} \mathbf{B}$. If we assume that the specific strain energy function depends on the symmetric strain tensor, $\psi=\psi_{s}\left(\mathbf{E}_{\mathrm{sym}}(\mathbf{E}), \mathbf{M}\right)$, we obtain

$$
\mathrm{D}_{\mathbf{E}} \psi_{s}\left(\mathbf{E}_{\mathrm{sym}}(\mathbf{E}), \mathbf{M}\right)[\mathbf{H}]=\mathrm{D}_{\mathbf{E}_{\mathrm{sym}}} \psi_{s}\left(\mathbf{E}_{\mathrm{sym}}, \mathbf{M}\right)\left[\mathrm{D}_{\mathbf{E}} \mathbf{E}_{\mathrm{sym}}(\mathbf{E})[\mathbf{H}]\right]=\overline{\mathcal{I}}^{T} \frac{\partial \psi}{\partial \mathbf{E}_{\mathrm{sym}}} \cdot \mathbf{H}
$$

i.e.,

$$
\begin{aligned}
\mathbf{T} & =\hat{\mathbf{h}}\left(\mathbf{E}_{\mathrm{sym}}(\mathbf{E}), \mathbf{M}\right)=\overline{\mathcal{I}}^{T} \frac{\partial \psi}{\partial \mathbf{E}_{\mathrm{sym}}}=\overline{\mathcal{I}}^{T} \underbrace{\mathbf{h}\left(\mathbf{E}_{\mathrm{sym}}(\mathbf{E}), \mathbf{M}\right)}_{\partial \psi / \partial \mathbf{E}_{\mathrm{sym}}} \\
& =\left(\Lambda \mathrm{I}_{\mathbf{E}_{\mathrm{sym}}}+\alpha \mathrm{IV}_{\mathbf{E}_{\mathrm{sym}}}\right) \mathbf{I}+2 \mu_{T} \mathbf{E}_{\mathrm{sym}}+\left(\alpha \mathrm{I}_{\mathbf{E}_{\mathrm{sym}}}+\beta \mathrm{IV}_{\mathbf{E}_{\mathrm{sym}}}\right) \mathbf{M}+2\left(\mu_{L}-\mu_{T}\right)\left(\mathbf{E}_{\mathrm{sym}} \mathbf{M}+\mathbf{M} \mathbf{E}_{\mathrm{sym}}\right)
\end{aligned}
$$

see [10] for "internal" and "external" differentials and the application of the chain rule. The tangent operator (fourth-order elasticity tensor) stems from

$$
\mathrm{D}_{\mathbf{E}} \hat{\mathbf{h}}\left(\mathbf{E}_{\mathrm{sym}}(\mathbf{E}), \mathbf{M}\right)[\mathbf{H}]=\overline{\mathcal{I}}^{T} \frac{\partial \mathbf{h}}{\partial \mathbf{E}_{\mathrm{sym}}} \overline{\mathcal{I}} \mathbf{H}=\mathcal{C}_{\mathrm{sym}} \mathbf{H}
$$

i.e.,

$$
\mathcal{C}_{\text {sym }}=\overline{\mathcal{I}}^{T} \frac{\partial \mathbf{h}}{\partial \mathbf{E}_{\mathrm{sym}}} \overline{\mathcal{I}}=\overline{\mathcal{I}}^{T} \mathcal{C} \overline{\mathcal{I}}
$$

To look at the results, a number of tensor products of fourth-order tensors and some transposition rules are required. The latter read

$$
\left[[\mathbf{A} \otimes \mathbf{B}]^{T_{23}}\right]^{T}=\left[\mathbf{A}^{T} \otimes \mathbf{B}^{T}\right]^{T_{23}}, \quad\left[[\mathbf{A} \otimes \mathbf{B}]^{T_{24}}\right]^{T}=[\mathbf{B} \otimes \mathbf{A}]^{T_{24}},
$$

where $[\ldots]^{T}$ means the exchange of the first and last two indices, $[\mathbf{A} \otimes \mathbf{B}]^{T}=\mathbf{B} \otimes \mathbf{A}$. The required products are

$$
\begin{aligned}
{[\mathbf{A} \otimes \mathbf{B}][\mathbf{C} \otimes \mathbf{D}]^{T_{23}} } & =\mathbf{A} \otimes\left[[\mathbf{C} \otimes \mathbf{D}]^{T_{23}}\right]^{T} \mathbf{B}=\mathbf{A} \otimes \mathbf{C}^{T} \mathbf{B D} \\
{[\mathbf{A} \otimes \mathbf{B}]^{T_{23}}[\mathbf{C} \otimes \mathbf{D}] } & =\left([\mathbf{A} \otimes \mathbf{B}]^{T_{23}} \mathbf{C}\right) \otimes \mathbf{D}=\mathbf{A} \mathbf{C} \mathbf{B}^{T} \otimes \mathbf{D} \\
{[\mathbf{A} \otimes \mathbf{B}]^{T_{23}}[\mathbf{C} \otimes \mathbf{D}]^{T_{23}} } & =[\mathbf{A C} \otimes \mathbf{B D}]^{T_{23}} \\
{[\mathbf{A} \otimes \mathbf{B}][\mathbf{C} \otimes \mathbf{D}]^{T_{24}} } & =\mathbf{A} \otimes\left[[\mathbf{C} \otimes \mathbf{D}]^{T_{24}}\right]^{T} \mathbf{B}=\mathbf{A} \otimes \mathbf{D B}^{T} \mathbf{C}
\end{aligned}
$$




$$
\begin{aligned}
{[\mathbf{A} \otimes \mathbf{B}]^{T_{24}}[\mathbf{C} \otimes \mathbf{D}] } & =\left([\mathbf{A} \otimes \mathbf{B}]^{T_{24}} \mathbf{C}\right) \otimes \mathbf{D}=\mathbf{A C}^{T} \mathbf{B} \otimes \mathbf{D} \\
{[\mathbf{A} \otimes \mathbf{B}]^{T_{24}}[\mathbf{C} \otimes \mathbf{D}]^{T_{24}} } & =\left[\mathbf{A} \mathbf{D}^{T} \otimes \mathbf{B}^{T} \mathbf{C}\right]^{T_{23}} \\
{[\mathbf{A} \otimes \mathbf{B}]^{T_{23}}[\mathbf{C} \otimes \mathbf{D}]^{T_{24}} } & =\left[\mathbf{A C} \otimes \mathbf{D} \mathbf{B}^{T}\right]^{T_{24}} \\
{[\mathbf{A} \otimes \mathbf{B}]^{T_{24}}[\mathbf{C} \otimes \mathbf{D}]^{T_{23}} } & =\left[\mathbf{A D} \otimes \mathbf{C}^{T} \mathbf{B}\right]^{T_{23}} .
\end{aligned}
$$

These relations are evaluated for the calculation of Eq. (97)

$$
\begin{aligned}
\overline{\mathcal{I}}^{T}[\mathbf{I} \otimes \mathbf{I}] \overline{\mathcal{I}} & =\mathbf{I} \otimes \mathbf{I} \\
\overline{\mathcal{I}}^{T} \mathcal{I} \overline{\mathcal{I}} & =\overline{\mathcal{I}} \\
\overline{\mathcal{I}}^{T}[\mathbf{I} \otimes \mathbf{M}+\mathbf{M} \otimes \mathbf{I}] \overline{\mathcal{I}} & =\mathbf{I} \otimes \mathbf{M}+\mathbf{M} \otimes \mathbf{I} \\
\overline{\mathcal{I}}^{T}[\mathbf{M} \otimes \mathbf{M}] \overline{\mathcal{I}} & =\mathbf{M} \otimes \mathbf{M} \\
\overline{\mathcal{I}}^{T}[\mathbf{I} \otimes \mathbf{M}+\mathbf{M} \otimes \mathbf{I}]^{T_{23}} \overline{\mathcal{I}} & =\frac{1}{2}\left[[\mathbf{I} \otimes \mathbf{M}+\mathbf{M} \otimes \mathbf{I}]^{T_{23}}+[\mathbf{I} \otimes \mathbf{M}+\mathbf{M} \otimes \mathbf{I}]^{T_{24}}\right] \\
& =\frac{1}{2}\left(a_{i}\left(\delta_{j k} a_{l}+\delta_{j l} a_{k}\right)+a_{j}\left(\delta_{i l} a_{k}+\delta_{i k} a_{l}\right)\right) \mathbf{e}_{i} \otimes \mathbf{e}_{j} \otimes \mathbf{e}_{k} \otimes \mathbf{e}_{l},
\end{aligned}
$$

where the latter can be found in [41] as well. Thus, we obtain

$\mathcal{C}_{\text {sym }}=\Lambda \mathbf{I} \otimes \mathbf{I}+2 \mu_{T} \overline{\mathcal{I}}+\alpha[\mathbf{I} \otimes \mathbf{M}+\mathbf{M} \otimes \mathbf{I}]+\beta \mathbf{M} \otimes \mathbf{M}+\left(\mu_{L}-\mu_{T}\right)\left[[\mathbf{I} \otimes \mathbf{M}+\mathbf{M} \otimes \mathbf{I}]^{T_{23}}+[\mathbf{I} \otimes \mathbf{M}+\mathbf{M} \otimes \mathbf{I}]^{T_{24}}\right]$

having the required major and minor symmetries.

The remaining question treats the subject whether different representations, $\mathcal{C}$ versus $\mathcal{C}_{\text {sym }}$, have different Voigt matrices. In the procedure defined, for example, in [15], to derive the Voigt matrix both matrices have the same entries for arbitrary vectors $\mathbf{a}$.

\section{References}

1. Al-Kinani, R., Netz, T., Hartmann, S.: Transversal isotropy based on a multiplicative decomposition of the deformation gradient within p-version finite elements. Z. Angew. Math. und Mech. 95, 742-761 (2015)

2. Andresen, K., Dannemeyer, S., Friebe, H., Mahnken, R., Ritter, R., Stein, E.: Parameteridentifikation für ein plastisches Stoffgesetz mit FE-Methoden und Rasterverfahren. Bauingenieur 71, 21-31 (1996)

3. Beck, J.V., Arnold, K.J.: Parameter Estimation in Engineering Science, 1st edn. Wiley, New York (1977)

4. Benedix, U., Görke, U.J., Kreißig, R., Kretzschmar, S.: Local and global analysis of inhomogeneous displacement fields for the identification of material parameters. In: Hoa SV, De Wilde WP, Blain WR (eds) Computer Methods in Composite Materials VI (CADCOMP 98), pp. 159-168 (1998)

5. Beveridge, G.S.G., Schechter, R.S.: Optimization: Theory and Practice, 1st edn. McGraw-Hill Book Company, New York (1970)

6. Chen, Z., Diebels, S.: Nanoindentation of soft polymers: modeling, experiments and parameter identification. Tech. Mech. 34, 166-189 (2014)

7. Christensen, R.M.: The numbers of elastic properties and failure parameters for fiber composites. J. Eng. Mater. Technol. 120, 110-113 (1998)

8. Christensen, R.M.: Mechanics of Composite Materials. Dover Publication, Mineoly (2005)

9. Cooreman, S., Lecompte, D., Sol, H., Vantomme, J., Debruyne, D.: Elasto-plastic material parameter identification by inverse methods: calculation of the sensitivity matrix. Int. J. Solids Struct. 44, 4329-4341 (2007)

10. Gurtin, M.E.: An Introduction to Continuum Mechanics. Academic Press, New York (1981)

11. Halpin, J.C., Kardos, J.L.: The halpin-tsai equations: a review. Polym. Eng. Sci. 16(5), 344-352 (1976)

12. Hartmann, S.: Numerical studies on the identification of the material parameters of Rivlin's hyperelasticity using tensiontorsion tests. Acta Mech. 148, 129-155 (2001)

13. Hartmann, S.: A remark on material parameter identification using finite elements based on constitutive models of evolutionary-type. Comput. Assist. Methods Eng. Sci. 24, 113-126 (2017)

14. Hartmann, S., Gilbert, R.R.: Identifiability of material parameters in solid mechanics. Arch. Appl. Mech. 88(1), 3-26 (2018)

15. Hartmann, S., Kheiri Marghzar, A.: Modeling of fiber circumplacement around a hole using a streamline approach. Univ. J. Math. Appl. 1, 17-28 (2018) 
16. Hartmann, S., Rodriguez, S.: Verification examples for strain and strain-rate determination of digital image correlation systems. In: Altenbach H, Jablonski F, Müller W, Naumenko K, Schneider P (eds) Advances in Mechanics of Materials and Structural Analysis. Advanced Structured Materials, no. 80 in Advanced Structured Materials, Springer International Publishing, Cham, pp. 135-174 (2018)

17. Hartmann, S., Tschöpe, T., Schreiber, L., Haupt, P.: Large deformations of a carbon black-filled rubber: experiment, optical measurement and parameter identification using finite elements. Eur. J. Mech. Ser. A/Solids 22, 309-324 (2003)

18. Hartmann, S., Gibmeier, J., Scholtes, B.: Experiments and material parameter identification using finite elements. Uniaxial tests and validation using instrumented indentation tests. Exp. Mech. 46(1), 5-18 (2006)

19. Hartmann, S., Quint, K.J., Hamkar, A.W.: Displacement control in time-adaptive non-linear finite-element analysis. ZAMM J. Appl. Math. Mech. 88(5), 342-364 (2008)

20. Hartmann, S., Gilbert, R.R., Sguazzo, C.: Basic studies in biaxial tensile tests. GAMM-Mitteilungen 41(e201800), 004 (2018)

21. Hartmann, S., Kheiri Marghzar, A., Gilbert, R.R., Pangboonyanon, W., Meiners, D.: Experiment, modeling and simulation of bypassing holes in composites. Compos. Struct. 234, 1-17 (2020). https://doi.org/10.1016/j.compstruct.2019.111666

22. Haupt, P.: Continuum Mechanics and Theory of Materials, 2nd edn. Springer, Berlin (2002)

23. Huber, N., Tsakmakis, C.: Determination of constitutive properties from spherical indentation data using neural networks, Part I: plasticity with nonlinear and kinematic hardening. J. Mech. Phys. Solids 47, 1589-1607 (1999a)

24. Huber, N., Tsakmakis, C.: Determination of constitutive properties from spherical indentation data using neural networks, Part II: the case of pure kinematic hardening in plasticity laws. J. Mech. Phys. Solids 47, 1569-1588 (1999b)

25. Itskov, M., Aksel, N.: A class of orthotropic and transversely isotropic hyperelastic constitutive models based on a polyconvex strain energy function. Int. J. Solids Struct. 41, 3833-3848 (2004)

26. Krämer, S.: Einfluss von Unsicherheiten in Materialparametern auf Finite-Elemente Simulationen. Phd-thesis, report no. 5/2016, Institute of Applied Mechanics, Clausthal University of Technology, Clausthal-Zellerfeld (2016)

27. Kreißig, R.: Auswertung inhomogener Verschiebungsfelder zur Identifikation der Parameter elastisch-plastischer Deformationsgesetze. Forsch. Ingenieurwes. 64, 99-109 (1998)

28. Kreissig, R., Benedix, U., Goerke, U.J.: Statistical aspects of the identification of material parameters for elasto-plastic models. Arch. Appl. Mech. 71, 123-134 (2001)

29. Lawson, C.L., Hanson, R.J.: Solving least squares problems. Siam Society for Industrial and Applied Mathematics, Philadelphia (1995)

30. Lee, H., Lee, J.H., Pharr, G.M.: A numerical approach to spherical indentation techniques for material property evaluation. J. Mech. Phys. Solids 53, 2037-2069 (2005)

31. Mahnken, R., Stein, E.: A unified approach for parameter identification of inelastic material models in the frame of the finite element method. Comput. Methods Appl. Mech. Eng. 136, 225-258 (1996)

32. Mallick, P.K.: Fiber-reinforced Composites, 3rd edn. CRC Press, Boca Raton (2008)

33. Miehe, C., Koch, A.: Computational micro-to-macro transitions of discretized microstructures undergoing small strains. Arch. Appl. Mech. 72, 300-317 (2002)

34. Nocedal, J., Wright, S.J.: Numerical Optimization. Springer, New York (1999)

35. Powell, M.: A direct search optimization method that models the objective and constraint functions by linear interpolations. In: Gomez, S., Hennart, J.P. (eds.) Advances in Optimization and Numerical Analysis, pp. 51-67. Kluwer Academic, Dordrecht (1994)

36. Powell, M.J.D.: Direct search algorithms for optimization calculations. Acta Numerica 7:287-336, (see internet address: http://plato.la.asu.edu/topics/problems/nlores.html) (1998)

37. Rauchs, G., Bardon, J., Georges, D.: Identification of the material parameters of a viscous hyperelastic constitutive law from spherical indentation tests of rubber and validation by tensile tests. Mech. Mater. 42, 961-973 (2010)

38. Scheday, G.: Theorie und Numerik der Parameteridentifikation von Materialmodellen der finiten Elastizität und Inelastizität auf der Grundlage optischer Feldmessmethoden. Phd-thesis, Report No. I-11 (2003), University of Stuttgart (Germany), Institute of Mechanics (2003)

39. Schenk, O., Gärtner, K.: On fast factorization pivoting methods for sparse symmetric indefinite systems. ETNA Electron. Trans. Numer. Anal. [electronic only] 23:158-179 (2006). http://eudml.org/doc/127439

40. Schittkowski, K.: Numerical Data Fitting in Dynamical Systems. Kluwer, Dordrecht (2002)

41. Schröder, J.: Theoretische und algorithmische Konzepte zur phänomenologischen Beschreibung anisotropen Materialverhaltens. Bericht i-1 1996, Universität Stuttgart (1996)

42. Schröder, J., Neff, P.: Invariant formulation of hyperelasticity transverse isotropy based on polyconvex free energy functions. Int. J. Solids Struct. 40, 401-445 (2003)

43. Sguazzo, C., Hartmann, S.: Tensile and shear experiments using polypropylene/polyethylene foils at different temperatures. Tech. Mech. 38, 166-190 (2018)

44. Spencer, A.J.M.: Constitutive theory for strongly anisotropic solids. In: Spencer AJM (ed) Continuum theory of the mechanics of fibre-reinforced composites, no. 282 in Courses and lectures - International Centre for Mechanical Sciences, Springer, Wien, pp. 1-32 (1984)

45. Taylor, J.R.: An Introduction to Error Analysis. University Science Books, California (1997)

46. Vannucci, P.: Anisotropic elasticity. Lecture Notes in Applied an Computational Mechanics, vol. 85, 1st edn. Springer Nature, Singapore (2018)

47. Weiss, J.A., Maker, B.N., Govindjee, S.: Finite element implementation of incompressible, transversely isotropic hyperelasticity. Comput. Methods Appl. Mech. Eng. 135, 107-128 (1996)

Publisher's Note Springer Nature remains neutral with regard to jurisdictional claims in published maps and institutional affiliations. 